\title{
Random graphs from a weighted minor-closed class
}

\author{
Colin McDiarmid \\ Department of Statistics \\ Oxford University, UK \\ cmcd@stats.ox.ac.uk
}

\begin{abstract}
Submitted: Oct 11, 2012; Accepted: Jun 1, 2013; Published: Jun 7, 2013
Mathematics Subject Classifications: 05C80, 05C30
\end{abstract}

\begin{abstract}
There has been much recent interest in random graphs sampled uniformly from the $n$-vertex graphs in a suitable minor-closed class, such as the class of all planar graphs. Here we use combinatorial and probabilistic methods to investigate a more general model. We consider random graphs from a 'well-behaved' class of graphs: examples of such classes include all minor-closed classes of graphs with 2-connected excluded minors (such as forests, series-parallel graphs and planar graphs), the class of graphs embeddable on any given surface, and the class of graphs with at most $k$ vertex-disjoint cycles. Also, we give weights to edges and components to specify probabilities, so that our random graphs correspond to the random cluster model, appropriately conditioned.

We find that earlier results extend naturally in both directions, to general wellbehaved classes of graphs, and to the weighted framework, for example results concerning the probability of a random graph being connected; and we also give results on the 2-core which are new even for the uniform (unweighted) case.
\end{abstract}

Keywords: random graph; minor-closed class; random cluster model; connectivity; core

\section{Introduction}

Given a class $\mathcal{A}$ of graphs (always assumed to be closed under isomorphism), let $\mathcal{A}_{n}$ denote the set of graphs in $\mathcal{A}$ on the vertex set $[n]:=\{1, \ldots, n\}$. There has been much recent interest in properties of the random graph $R_{n}$ sampled uniformly from $\mathcal{A}_{n}$, when $\mathcal{A}$ is a suitable 'structured' class of graphs such as the class of all planar graphs.

Analytic methods, based on generating functions and singularity analysis, have over recent years been extended dramatically to handle more and more complicated classes 
of graphs: the work on planar graphs by Giménez and Noy [35] (see also [4, 36]) was a breakthrough, and very recently graphs embeddable on any given surface have been handled [3, 17]. See also [31] for graphs with no minor isomorphic to the complete bipartite graph $K_{3,3}$. Analytic work in papers such as those just mentioned much extends earlier combinatorial and probabilistic investigations, as for example in [32, 33, 34, 47, 48, 51, $52,53]$. For further recent related work (appearing in 2010 or later) see for example $[6,10,16,20,21,22,23,24,25,26,29,41,42,43,44,49,56,57]$.

There is natural interest also in the case when $\mathcal{A}$ is any minor-closed class of graphs, or for example any such class with 2-connected excluded minors; and for such investigations we still need combinatorial and probabilistic methods. Here we use such methods, building in particular on [48], and consider a more general model: we investigate random graphs from a suitable weighted class of graphs, where 'suitable' includes all the usual suspects and more, and 'weighted' is described below, corresponding to the random clusster model.

\subsection{The model}

To introduce the model, recall that in the classical binomial random graph $G_{n, p}$ on the vertex set $[n]$, the $\left(\begin{array}{l}n \\ 2\end{array}\right)$ possible edges are included independently with probability $p$, where $0<p<1$, see for example [14,39]. Assuming that $\mathcal{A}_{n}$ is non-empty, for each $H \in \mathcal{A}_{n}$ we have

$$
\mathbb{P}\left(G_{n, p}=H \mid G_{n, p} \in \mathcal{A}\right)=\frac{p^{e(H)}(1-p)^{\left(\begin{array}{c}
n \\
2
\end{array}\right)-e(H)}}{\sum_{G \in \mathcal{A}_{n}} p^{e(G)}(1-p)^{\left(\begin{array}{l}
n \\
2
\end{array}\right)-e(G)}}=\frac{\lambda^{e(H)}}{\sum_{G \in \mathcal{A}_{n}} \lambda^{e(G)}}
$$

where $\lambda=p /(1-p)$. Here $e(G)$ denotes the number of edges in $G$ : we will use $v(G)$ similarly to denote the number of vertices in $G$.

Now consider the more general random-cluster model, see for example [38], where we are also given a parameter $\nu>0$; and the random graph $R_{n}$ takes as values the graphs $H$ on $[n]$, with

$$
\mathbb{P}\left(R_{n}=H\right) \propto p^{e(H)}(1-p)^{\left(\begin{array}{l}
n \\
2
\end{array}\right)-e(H)} \nu^{\kappa(H)} .
$$

Here $\kappa(H)$ denotes the number of components of $H$. For each $H \in \mathcal{A}_{n}$ we have

$$
\mathbb{P}\left(R_{n}=H \mid R_{n} \in \mathcal{A}\right)=\frac{\lambda^{e(H)} \nu^{\kappa(H)}}{\sum_{G \in \mathcal{A}_{n}} \lambda^{e(G)} \nu^{\kappa(G)}} .
$$

This is the distribution on which we shall focus. Thus the distribution of our random graphs in $\mathcal{A}$ is as follows. Given edge-parameter $\lambda>0$ and component-parameter $\nu>0$, we let the weighting $\tau$ be the pair $(\lambda, \nu)$. For each graph $G$ we let $\tau(G)=\lambda^{e(G)} \nu^{\kappa(G)}$; and for each finite set $\mathcal{B}$ of graphs we denote $\sum_{G \in \mathcal{B}} \tau(G)$ by $\tau(\mathcal{B})$. We write $R \in_{\tau} \mathcal{B}$ to indicate that $R$ is a random graph which takes values in $\mathcal{B}$ with

$$
\mathbb{P}(R=H)=\frac{\tau(H)}{\tau(\mathcal{B})}
$$


and we call $R$ a $\tau$-weighted random graph from $\mathcal{B}$. Given a fixed weighted graph class $(\mathcal{A}, \tau)$, we write $R_{n} \in_{\tau} \mathcal{A}$ to indicate that $R_{n}$ has the distribution of $R \in_{\tau} \mathcal{A}_{n}$ (when $\mathcal{A}_{n}$ is non-empty).

Our aim is to investigate the behaviour of the $\tau$-weighted random graph $R_{n}$ for a suitable weighted graph class $(\mathcal{A}, \tau)$, with a fixed $\tau$, for large $n$. When $\lambda=\nu=1$ of course we are back to random graphs sampled uniformly. Let us write $R_{n} \in_{u} \mathcal{A}$ to indicate that $R_{n}$ is uniformly distributed over $\mathcal{A}_{n}$, as introduced in [53] (and perhaps earlier).

Analytic methods for graph problems often involve generating functions with a variable $x$ for vertices and a variable $y$ for edges (and sometimes a variable $z$ for components), as for example in [35]; and we may think of $y$ as giving a weight for edges. Also for example, for a fixed $1<\mu<3$, we may learn about the random planar graph with $n$ vertices and with $\sim \mu n$ edges by choosing a suitable value for the edge-weight, see [35]. Further, models in physics involving lattices or more general graphs may attach weights to vertices or edges; for example the hard-core model, which is a model for a gas with particles of non-negligible size, and which also appears in models for communications networks, see for example [5].

We find that many results extend naturally from the uniform case $\tau=(1,1)$ to general $\tau$-weighted random graphs. As well as generalising previous work on the uniform case in this way, we give new results on the 2-core of $R_{n}$, arising from a more combinatorial proof of a key 'smoothness' result, see $[2,48]$. The 2-core of a graph $G$ (sometimes called just the core), denoted here by $2-\operatorname{core}(G)$, is the unique maximal subgraph with minimum degree is at least 2. Thus $2-\operatorname{core}(G)$ is empty if and only if $G$ is a forest; and the 2-core may be obtained by repeatedly trimming off leaves.

To investigate the random graph $R_{n} \in_{\tau} \mathcal{A}$ we need to consider how $\tau\left(\mathcal{A}_{n}\right)$ grows with $n$. As in the uniform case, we say that the weighted graph class $(\mathcal{A}, \tau)$ has growth constant $\gamma=\gamma(\mathcal{A}, \tau)$ if $0 \leqslant \gamma<\infty$ and $\tau\left(\mathcal{A}_{n}\right)=(\gamma+o(1))^{n} n$ ! as $n \rightarrow \infty$. (Sometimes we insist that $\gamma>0$.) Also we say that $(\mathcal{A}, \tau)$ is smooth (or smoothly growing) if $\tau\left(\mathcal{A}_{n}\right) / n \tau\left(\mathcal{A}_{n-1}\right)$ tends to a limit $\gamma^{\prime}$ with $0<\gamma^{\prime}<\infty$ as $n \rightarrow \infty$. (This is also referred to as the 'ratio test' property RT1, see for example [1, 15].) It is easy to see that in this case the limit $\gamma^{\prime}$ must be the growth constant $\gamma$.

We need some definitions concerning a graph class $\mathcal{A}$. We say that $\mathcal{A}$ is proper when it is not the class of all graphs and it contains a graph with at least one edge; that $\mathcal{A}$ is decomposable when a graph is in $\mathcal{A}$ if and only if each component is; that $\mathcal{A}$ is bridge-addable when, for each graph in $\mathcal{A}$ and each pair $u$ and $v$ of vertices in different components, the graph obtained by adding an edge joining $u$ and $v$ must also be in $\mathcal{A}$; and $\mathcal{A}$ is addable when it is both decomposable and bridge-addable. Also, we say that $\mathcal{A}$ is minor-closed if whenever $G \in \mathcal{A}$ and $H$ is a minor of $G$ then $H \in \mathcal{A}$.

Let $\mathcal{A}$ be a proper minor-closed class of graphs. The minor-minimal graphs not in $\mathcal{A}$ are the excluded minors. From the Robertson and Seymour theory of graph minors [58], see for example Diestel [19], the set of excluded minors must be finite. The properties of being decomposable and being addable correspond to simple properties of the excluded minors: indeed, $\mathcal{A}$ is decomposable if and only if each excluded minor is connected, and 
$\mathcal{A}$ is addable if and only if each excluded minor is 2-connected.

It was conjectured in [6] that, in the uniform case, every proper minor-closed class of graphs has a growth constant. It is natural to conjecture that this in fact holds for each weighting $\tau$. Indeed it is natural to conjecture that we even have smoothness, whenever the growth constant is $>0$.

\subsection{Overview of main results}

Our results involve a 'well-behaved' weighted class of graphs. We shall say later precisely what this means, after we have introduced various preliminary definitions. We require that such a class is proper, minor-closed and bridge-addable, and satisfies certain further conditions: the full definition is given in section 2.5.4 below. The important thing to note here is that the following classes of graphs are all well-behaved, with any weighting $\tau$ : any proper, minor-closed, addable class (for example the class of forests, or series-parallel graphs or planar graphs); the class $\mathcal{G}^{S}$ of graphs embeddable on any given surface $S$; and the class of all graphs which contain at most $k$ vertex-disjoint cycles, for some fixed $k$.

We shall prove various results about any well-behaved weighted class of graphs $(\mathcal{A}, \tau)$ and about the corresponding random graph $R_{n} \in_{\tau} \mathcal{A}$, where $\lambda>0, \nu>0$ and $\tau=(\lambda, \nu)$. We sketch some of these results now, in the order in which the proofs run: full details appear in Section 2.

First we show the key counting result that $(\mathcal{A}, \tau)$ is smooth, with some growth constant $\gamma>0$ (depending on $\mathcal{A}$ and $\lambda$ but not on $\nu$ ). In the process of doing this we learn about the 2-core of $R_{n}$ : in particular we find that, as $n \rightarrow \infty$

$$
v\left(2-\operatorname{core}\left(R_{n}\right)\right) / n \rightarrow(1-t) \text { in probability }
$$

where $t$ is the unique root with $0<t<1$ to $e^{t} / t=\lambda \gamma$. When $\mathcal{A}$ is the class $\mathcal{G}^{S}$ of graphs embeddable on a given surface $S$ and $\lambda=1$ then from [35,47] we have $\gamma \approx 27.22687$, and so $v\left(2-\operatorname{core}\left(R_{n}\right)\right) \approx 0.96184 n$ whp. (We say that a sequence $\left(A_{n}\right)$ of events holds with high probability (whp ) if $\operatorname{Pr}\left(A_{n}\right) \rightarrow 1$ as $n \rightarrow \infty$.)

After that, using smoothness, we learn about the 'fragments' of $R_{n}$ not in the giant component, and in particular we find that

$$
\mathbb{P}\left(R_{n} \text { is connected }\right) \rightarrow e^{-D(\rho, \tau)}=e^{-\nu D\left(\rho, \tau_{1}\right)} \text { as } n \rightarrow \infty
$$

where $\tau_{1}=(\lambda, 1)$ and $D(\rho, \tau)$ is as described in the next paragraph. When $\mathcal{A}$ is the class of forests, the growth constant $\gamma$ is $e \lambda$ and the limiting probability of connectedness is $e^{-\frac{\nu}{2 \lambda}}$. When $\mathcal{A}$ is the class $\mathcal{G}^{S}$ of graphs embeddable on a given surface $S$ and $\lambda=1$ the limiting probability of connectedness is $e^{-\nu D(\rho, \mathbf{1})} \approx 0.96325^{\nu}$ (see Section 2.3).

We say that a graph $H$ is freely addable to a graph class $\mathcal{A}$ if the disjoint union $G \cup H$ of $G$ and $H$ is in $\mathcal{A}$ for each graph $G \in \mathcal{A}$. Let $\mathcal{D}$ denote the class of connected graphs which are freely addable to $\mathcal{A}$. Observe that if $\mathcal{A}$ is decomposable then each graph in $\mathcal{A}$ is freely addable to $\mathcal{A}$, so that $\mathcal{D}$ is the class of connected graphs in $\mathcal{A}$; and if $\mathcal{A}=\mathcal{G}^{S}$ then $\mathcal{D}$ is the class of connected planar graphs. Now $D(\rho, \tau)$ is the evaluation of the exponential generating function $D(x, \tau)$ for $\mathcal{D}$ (see Subsection 2.1), at the radius of convergence $\rho$ for $(\mathcal{A}, \tau)$. 


\section{Statement of main results}

The first subsection describes the Boltzmann Poisson random graph corresponding to a decomposable class. Then we consider a well-behaved weighted class $(\mathcal{A}, \tau)$ of graphs and $R_{n} \in_{\tau} \mathcal{A}$. We describe how the 'fragments' not in the giant component of $R_{n}$ converge in distribution to $R$, for the corresponding Boltzmann Poisson random graph $R$, which gives as a corollary the result (2) on the limiting probability of $R_{n}$ being connected. The next subsection concerns smoothness and the 2-core, and in particular includes the result (1); and then we discuss appearances of subgraphs. In the final subsections we say precisely what it means for a graph class to be 'well-behaved', and then give a sketch plan of the rest of the paper.

\subsection{Boltzmann Poisson random graph}

We introduce a general distribution on the unlabelled graphs corresponding to a weighted class of labelled graphs.

Let $\mathcal{A}$ be a class of graphs, and let $\mathcal{C}$ be the class of connected graphs in $\mathcal{A}$. (By convention the empty graph $\emptyset$ is in $\mathcal{A}$ and not in $\mathcal{C}$.) We define the generating function $A(x, y, z)$ by

$$
A(x, y, z)=\sum_{n \geqslant 0} \sum_{G \in \mathcal{A}_{n}} \frac{x^{n}}{n !} y^{e(G)} z^{\kappa(G)}
$$

and similarly

$$
C(x, y, z)=z \sum_{n \geqslant 1} \sum_{G \in \mathcal{C}_{n}} \frac{x^{n}}{n !} y^{e(G)} .
$$

The standard 'exponential formula' in a general form (see for example $[28,59]$ ) is that when $\mathcal{A}$ is decomposable we have

$$
A(x, y, z)=e^{C(x, y, z)} .
$$

Let us say that $\mathcal{A}$ contains components (or is down-decomposable) if each component of each graph in $\mathcal{A}$ is also in $\mathcal{A}$. Suppose that $\mathcal{A}$ contains components, and consider any fixed positive $y$ and $z$. Then as in $(3), C(x, y, z) \leqslant A(x, y, z) \leqslant e^{C(x, y, z)}$ for each $x \geqslant 0$, and so the generating functions $A(x, y, z)$ and $C(x, y, z)$ (as functions of $x$ ) have the same radius of convergence. Thus in particular the radius of convergence of $A$ does not depend on $z$. (The radius of convergence clearly depends on $y$ since $e(G)$ is at least linear for a connected graph $G$.) Also we may see that $(\mathcal{A}, \tau)$ has growth constant $\gamma$ if and only if $(\mathcal{C}, \tau)$ does.

For any graph class $\mathcal{A}$, we let $\mathcal{U} \mathcal{A}$ denote the corresponding unlabelled graph class, with members the equivalence classes of graphs in $\mathcal{A}$ under isomorphism. Now let $\mathcal{A}$ be any decomposable class of (labelled) graphs. As we shall observe later, we may write its generating function $A(x, y, z)$ in terms of $\mathcal{U} \mathcal{A}$ as

$$
A(x, y, z)=\sum_{H \in \mathcal{U} \mathcal{A}} \frac{x^{v(H)} y^{e(H)} z^{\kappa(H)}}{\operatorname{aut}(H)} .
$$


Here aut $(G)$ denotes the number of automorphisms of $G$. Suppose that we are given $\tau=(\lambda, \nu)$. We shall set $y=\lambda$ and $z=\nu$, and write either $A(x, \lambda, \nu)$ or $A(x, \tau)$. If we choose $\rho>0$ such that $A(\rho, \tau)$ is finite, then we may obtain a natural 'Boltzmann Poisson distribution' on $\mathcal{U} \mathcal{A}$ - see equation (6) below. The uniform case $\tau=(1,1)$ was considered in [48]. We denote the radius of convergence of $A(x, \tau)$ (viewed as a power series in $x$ ) by $\rho(\mathcal{A}, \tau)$.

We need more notation (following [48]) to record some of the properties of this distribution. For a connected graph $H$ let $\kappa(G, H)$ denote the number of components of $G$ isomorphic to $H$; and for a class $\mathcal{D}$ of connected graphs let $\kappa(G, \mathcal{D})$ denote $\sum_{H \in \mathcal{U D}} \kappa(G, H)$, the number of components of $G$ isomorphic to some graph in $\mathcal{D}$. The notation $X \sim \operatorname{Po}(\mu)$ means that the random variable $X$ has the Poisson distribution with mean $\mu$. Recall that a sum of independent Poisson random variables $\operatorname{Po}\left(\mu_{i}\right)$ has distribution $\operatorname{Po}\left(\sum_{i} \mu_{i}\right)$, as long as $\sum_{i} \mu_{i}<\infty$.

Theorem 2.1. Consider the weighted graph class $(\mathcal{A}, \tau)$ where $\mathcal{A}$ is decomposable. Let $\rho>0$ be such that $A(\rho, \tau)$ is finite, and let

$$
\mu(H)=\frac{\rho^{v(H)} \lambda^{e(H)} \nu^{\kappa(H)}}{\operatorname{aut}(H)} \text { for each } H \in \mathcal{U} \mathcal{A}
$$

(so that $A(\rho, \tau)=\sum_{H \in \mathcal{U A}} \mu(H)$ by equation (4)). Let the 'Boltzmann Poisson random graph' $R=R(\mathcal{A}, \rho, \tau)$ take values in $\mathcal{U} \mathcal{A}$, with

$$
\mathbb{P}[R=H]=\frac{\mu(H)}{A(\rho, \tau)} \quad \text { for each } H \in \mathcal{U} \mathcal{A}
$$

Also, let $\mathcal{C}$ denote the class of connected graphs in $\mathcal{A}$.

Then the random variables $\kappa(R, H)$ for $H \in \mathcal{U C}$ are independent, with $\kappa(R, H) \sim$ $\operatorname{Po}(\mu(H))$.

In particular, since $C(\rho, \tau)=\sum_{H \in \mathcal{U C}} \mu(H)$ (by equation (4) applied to $\mathcal{C}$ ) we have $\kappa(R) \sim \operatorname{Po}(C(\rho, \tau))$.

\subsection{Fragments and connectivity}

The big component $\operatorname{Big}(G)$ of a graph $G$ is the (lexicographically first) component with the most vertices, and $\operatorname{Frag}(G)$ is the fragments subgraph induced on the vertices not in the big component (thus $\operatorname{Frag}(G)$ may be empty). Denote the numbers of vertices in $\operatorname{Big}(G)$ and $\operatorname{Frag}(G)$ by $\operatorname{big}(G)$ and $\operatorname{frag}(G)$ respectively, so $\operatorname{big}(G)+\operatorname{frag}(G)=v(G)$. We consider $R_{n} \in_{\tau} \mathcal{A}$, and focus on the limiting distribution of the random $\operatorname{graph} \operatorname{Frag}\left(R_{n}\right)$. It is convenient to deal with the random unlabelled graph $F_{n}$ corresponding to $\operatorname{Frag}\left(R_{n}\right)$. We use $\rightarrow_{T V}$ to denote convergence in total variation (or in distribution).

Theorem 2.2. Let the weighted graph class $(\mathcal{A}, \tau)$ be well-behaved, and let $\rho=\rho(\mathcal{A}, \tau)$. Let $\mathcal{F}_{\mathcal{A}}$ be the class of graphs freely addable to $\mathcal{A}$, with exponential generating function 
$F_{\mathcal{A}}$. Then $0<\rho<\infty$ and $F_{\mathcal{A}}(\rho, \tau)$ is finite; and for the random graph $R_{n} \in_{\tau} \mathcal{A}$, the random unlabelled graph $F_{n}$ corresponding to $\operatorname{Frag}\left(R_{n}\right)$ satisfies $F_{n} \rightarrow_{T V} R$, where $R$ is the Boltzmann Poisson random graph for $\mathcal{F}_{\mathcal{A}}, \rho, \tau$ defined in (6). Further, $\mathbb{E}[v(R)]=$ $\rho D^{\prime}(\rho, \tau)<\infty$, where $\mathcal{D}$ is the class of connected graphs in $\mathcal{F}_{\mathcal{A}}$.

Corollary 2.3. (a) For any given distinct graphs $H_{1}, \ldots, H_{k}$ in $\mathcal{U D}$ the $k$ random variables $\kappa\left(F_{n}, H_{i}\right)$ are asymptotically independent with distribution $\operatorname{Po}\left(\mu\left(H_{i}\right)\right)$.

(b) For any class $\tilde{\mathcal{D}} \subseteq \mathcal{D}$ we have $\kappa\left(F_{n}, \tilde{\mathcal{D}}\right) \rightarrow_{T V} \operatorname{Po}(\tilde{D}(\rho, \tau))$, and each moment of $\kappa\left(F_{n}, \tilde{\mathcal{D}}\right)$ tends to that of $\operatorname{Po}(\tilde{D}(\rho, \tau))$.

(c) As a special case of part (b), $\kappa\left(F_{n}\right) \rightarrow_{T V} 1+\operatorname{Po}(D(\rho, \tau))$, and as $n \rightarrow \infty$ we have $\mathbb{P}\left[F_{n}\right.$ is connected $] \rightarrow e^{-D(\rho, \tau)}=F_{\mathcal{A}}(\rho, \tau)^{-1}, \mathbb{E}\left[\kappa\left(F_{n}\right)\right] \rightarrow 1+D(\rho, \tau)$, and the variance of $\kappa\left(F_{n}\right)$ tends to $D(\rho, \tau)$.

(d) For the random number of vertices $v\left(F_{n}\right)=\operatorname{frag}\left(R_{n}\right)$, we have $v\left(F_{n}\right) \rightarrow_{T V} v(R)$; that is, for each non-negative integer $k$

$$
\mathbb{P}\left[v\left(F_{n}\right)=k\right] \rightarrow \frac{1}{F_{\mathcal{A}}(\rho, \tau)} \frac{\rho^{k}}{k !} \tau\left(\left(\mathcal{F}_{\mathcal{A}}\right)_{k}\right) \text { as } n \rightarrow \infty,
$$

and similarly $e\left(F_{n}\right) \rightarrow_{T V} e(R)$.

In the uniform case $\tau=(1,1)$ of the above result, part (c) on $\kappa\left(R_{n}\right)$ and part (d) on $v\left(F_{n}\right)$ extend for example Theorems 5.2 and 5.3 of [17].

Trees and forests Let us illustrate the above results for the classes $\mathcal{T}$ of trees and $\mathcal{F}$ of forests. Denote $\mathcal{F}$ by $\mathcal{A}$ temporarily (just the next three times). The class $\mathcal{A}$ is minorclosed and addable, and so it is well-behaved; and the class $\mathcal{F}_{\mathcal{A}}$ of graphs freely addable to $\mathcal{A}$ is just $\mathcal{F}$ again.

By Cayley's formula $\tau\left(\mathcal{T}_{n}\right)=n^{n-2} \lambda^{n-1} \nu$, and by Stirling's formula $(n !)^{1 / n} \sim n / e$. Thus $\left(\tau\left(\mathcal{T}_{n}\right) / n !\right)^{1 / n} \rightarrow e \lambda$ as $n \rightarrow \infty$, so that $(\mathcal{T}, \tau)$ has growth constant $e \lambda$. By Lemma 3.1 below

$$
\tau(\mathcal{F}, \tau) \geqslant \tau(\mathcal{T}, \tau) \geqslant e^{-\nu / \lambda} \tau(\mathcal{F}, \tau)
$$

and it follows that $(\mathcal{F}, \tau)$ also has growth constant $e \lambda$ (see also Section 3.7 below). Thus $\rho=\rho(\mathcal{F}, \tau)=(e \lambda)^{-1}$. Recall that

$$
\sum_{n \geqslant 1} \frac{n^{n-2}}{e^{n} n !}=\frac{1}{2} \quad \text { and } \quad \sum_{n \geqslant 1} \frac{n^{n-1}}{e^{n} n !}=1 .
$$

(One way to see these results is to consider the exponential generating functions $U(z)$ for (Cayley) trees and $T(z)$ for rooted trees respectively, where $U(z)=\sum_{n \geqslant 1} n^{n-2} z^{n} / n$ ! and $T(z)=\sum_{n \geqslant 1} n^{n-1} z^{n} / n$ !. Since $T(z)=z e^{T(z)}$ we find $T(1 / e)=1$, and since $U(z)=$ $T(z)-T^{2}(z) / 2$ we find $U(1 / e)=1 / 2$, see for example Stanley [59] chapter 5 , or Flajolet and Sedgewick [28] section II.5.).

Thus the exponential generating function $T$ (for the weighted case) satisfies

$$
T(\rho, \tau)=\nu \sum_{n \geqslant 1} n^{n-2} \lambda^{n-1}(e \lambda)^{-n} / n !=\frac{\nu}{\lambda} \sum_{n \geqslant 1} \frac{n^{n-2}}{e^{n} n !}=\frac{\nu}{2 \lambda} .
$$


and

$$
T^{\prime}(\rho, \tau)=\nu \sum_{n \geqslant 1} n^{n-1} \lambda^{n-1}(e \lambda)^{-(n-1)} / n !=e \nu \sum_{n \geqslant 1} \frac{n^{n-2}}{e^{n} n !}=e \nu .
$$

Now consider $R_{n} \in_{\tau} \mathcal{F}$. It follows from Corollary 2.3 part (c) that, as $n \rightarrow \infty, \kappa\left(R_{n}\right)$ converges in distribution to $1+\operatorname{Po}(\nu / 2 \lambda)$, and so in particular

$$
\mathbb{P}\left(R_{n} \text { is connected }\right)=\frac{\tau\left(\mathcal{T}_{n}\right)}{\tau\left(\mathcal{F}_{n}\right)} \rightarrow e^{-\frac{\nu}{2 \lambda}}
$$

and so

$$
\tau\left(\mathcal{F}_{n}\right) \sim \nu e^{\frac{\nu}{2 \lambda}} n^{n-2} \lambda^{n-1}
$$

Also, by part (d), as $n \rightarrow \infty, \operatorname{Frag}\left(R_{n}\right)$ converges in distribution to $R$, so $\operatorname{frag}\left(R_{n}\right)$ converges in distribution to $v(R)$, and indeed in this case we may see that also $\mathbb{E}\left[\operatorname{frag}\left(R_{n}\right)\right] \rightarrow$ $\mathbb{E}[v(R)]$ as $n \rightarrow \infty$ (this follows using the formulae above for $\tau\left(\mathcal{T}_{n}\right)$ and $\tau\left(\mathcal{F}_{n}\right)$, and arguing as in the proof of Proposition 5.2 of [47]), where by Theorem $2.2 \mathbb{E}[v(R)]=\rho T^{\prime}(\rho, \tau)=\frac{\nu}{\lambda}$.

Now consider (vertex-) rooted trees, which also have growth constant $e \lambda$. The exponential generating function $T^{o}$ satisfies

$$
T^{o}\left((e \lambda)^{-1}, \tau\right)=\nu \sum_{n \geqslant 1} n^{n-1} \lambda^{n-1}(e \lambda)^{-n} / n !=\frac{\nu}{\lambda} \sum_{n \geqslant 1} \frac{n^{n-1}}{e^{n} n !}=\frac{\nu}{\lambda},
$$

where we have used (7). This result is used in the introduction to Section 5.2.

\subsection{Smoothness and $2-\operatorname{core}\left(R_{n}\right)$}

Our next theorem says that a well-behaved weighted graph class $(\mathcal{A}, \tau)$ is smooth, and gives results on $2-\operatorname{core}\left(R_{n}\right)$ for the random graph $R_{n} \in_{\tau} \mathcal{A}$. Recall that $(\mathcal{F}, \tau)$ has growth constant $\lambda e$, where $\mathcal{F}$ is the class of forests.

Theorem 2.4. Let the weighted graph class $(\mathcal{A}, \tau)$ be well-behaved, with growth constant $\gamma$; let $\mathcal{C}$ denote the class of connected graphs in $\mathcal{A}$; and let $R_{n} \in_{\tau} \mathcal{A}$. Then

(a) Both $(\mathcal{A}, \tau)$ and $(\mathcal{C}, \tau)$ are smooth with growth constant $\gamma$, and $\gamma \geqslant \lambda e$.

(b) Let $\mathcal{B}$ denote the class $\mathcal{C}^{\delta \geqslant 2}$ of graphs in $\mathcal{C}$ with minimum degree at least 2. If $\gamma>\lambda e$ then $(\mathcal{B}, \tau)$ has growth constant $\beta$ where $\beta$ is the unique root $>\lambda$ to $\beta e^{\lambda / \beta}=\gamma$; and if $\gamma \leqslant$ de then $\rho(\mathcal{B}, \tau) \geqslant \lambda^{-1}$.

(c) If $\gamma>\lambda$ e let $\alpha=1-x$ where $x$ is the unique root $<1$ to $x e^{-x}=\lambda / \gamma$; and otherwise let $\alpha=0$. Then for each $\epsilon>0$

$$
\mathbb{P}\left(\left|v\left(2-\operatorname{core}\left(R_{n}\right)\right)-\alpha n\right|>\epsilon n\right)=e^{-\Omega(n)} .
$$


(d) Let $\mathcal{T}$ denote the class of trees and let $\mathcal{D}$ denote the class of connected graphs which are freely addable to $\mathcal{A}$, with generating functions $T$ and $D$ respectively; and let $\rho=1 / \gamma$. Suppose that $\gamma>\lambda e$ (so the probability that $2-\operatorname{core}\left(R_{n}\right)$ is empty is $e^{-\Omega(n)}$ by part $(c))$. Then $T(\rho, \tau)<D(\rho, \tau)<\infty$, and the probability that 2 - $\operatorname{core}\left(R_{n}\right)$ is non-empty and connected tends to $e^{T(\rho, \tau)-D(\rho, \tau)}$ as $n \rightarrow \infty$.

Graphs on surfaces Let us illustrate the theorem above for the class $\mathcal{G}^{S}$ of graphs embeddable on a given surface $S$. It was shown in [47] that for any fixed surface $S$, the class $\mathcal{G}^{S}$ of graphs embeddable on $S$ has growth constant $\gamma$, where $\gamma$ is the planar graph growth constant (the same $\gamma$ for each surface), and recently this was very much improved to give an asymptotic formula for $\left|\mathcal{G}_{n}^{S}\right|$, see [17, 3]. From Giménez and Noy [35] we have $\gamma \approx 27.226878$. For any weighting $\tau$, the weighted class $\left(\mathcal{G}^{S}, \tau\right)$ is well-behaved (this is part of lemma 2.7 below), and so by Theorem 2.4 we see that $\left(\mathcal{G}^{S}, \tau\right)$ is smooth; and when we specialise to the uniform case we obtain the result of [2] that the (uniform) class $\mathcal{G}^{S}$ is smooth (this also follows directly from the recent asymptotic formula for $\left|\mathcal{G}_{n}^{S}\right|$ mentioned above). Further we obtain new information on the core of a uniform random graph $R_{n} \in \mathcal{G}^{S}$, as follows.

Solving $\beta e^{1 / \beta}=\gamma$ (using the more accurate figure for $\gamma$ in Theorem 1 in [35]) gives $\beta=\beta_{0} \approx 26.207554$, and solving $\alpha=1-1 / \beta$ gives $\alpha=\alpha_{0} \approx 0.961843$. Thus the class $\mathcal{B}$ of (connected) graphs in $\mathcal{G}^{S}$ with minimum degree at least 2 has growth constant $\beta_{0}$ and $v\left(2-\operatorname{core}\left(R_{n}\right)\right) \approx \alpha_{0} n$ whp. The growth constant $\beta_{0}$ for (connected) graphs in $\mathcal{G}^{S}$ with each degree at least 2 is only slightly larger than the growth constant $\approx 26.18412$ for 2-connected graphs in $\mathcal{G}^{S}$, from $[4,35]$. Also, the asymptotic number $\alpha_{0} n$ of vertices in the core of $R_{n}$ is only slightly larger than the number of vertices in the largest block, which is about $0.95982 n$ by Theorem 5.4 of [37].

Finally, let us consider the connectivity of the 2-core. The class $\mathcal{D}$ of connected freely addable graphs is the class of all connected planar graphs, and from Corollary 1 in [35] we have $e^{-D(\rho)} \approx 0.963253$, where $\rho=1 / \gamma$. Further $e^{T(\rho)} \approx 1.038138$, so by Theorem 2.4 part $(\mathrm{d})$ the probability that $2-\operatorname{core}\left(R_{n}\right)$ is connected $\approx 0.999990$ (for large $n$ ). Thus the probability that $2-\operatorname{core}\left(R_{n}\right)$ is not connected $\approx 10^{-5}$. For comparison note that $\mathbb{P}\left(\operatorname{Frag}\left(R_{n}\right)=C_{3}\right) \sim e^{-D(\rho)} \rho^{3} / 6 \approx 8 \cdot 10^{-6}$.

\subsection{Appearances theorem}

It is often useful to know that for $R_{n} \in_{\tau} \mathcal{A}$, whp $R_{n}$ contains many disjoint copies of a given connected graph $H$. Suppose that $H$ has a specified root vertex $r$. We say that $H$ is freely attachable to $\mathcal{A}$ if, given any graph $G \in \mathcal{A}$ and vertex $v \in G$, the graph formed from the disjoint union $G \cup H$ by adding the edge $v r$ is in $\mathcal{A}$.

Let $H$ be a graph on the vertex set $[h]=\{1, \ldots, h\}$, and let $G$ be a graph on the vertex set $[n]$ where $n>h$. Let $W \subset V(G)$ with $|W|=h$, and let the root $r_{W}$ be the least element in $W$. We say that $H$ has a pendant appearance at $W$ in $G$ if (a) the increasing bijection from $[h]$ to $W$ gives an isomorphism between $H$ and the induced subgraph $G[W]$ of $G$; and (b) there is exactly one edge in $G$ between $W$ and the rest of $G$, and this edge is incident with the root $r_{W}$. We let $f_{H}(G)$ be the number of pendant appearances of $H$ 
in $G$, that is the number of sets $W \subseteq V(G)$ such that $H$ has a pendant appearance at $W$ in $G$. The next theorem extends results in $[52,53,47,48]$.

Theorem 2.5. Let the weighted graph class $(\mathcal{A}, \tau)$ have growth constant $\gamma$, and let $R_{n} \in_{\tau}$ $\mathcal{A}$. Let the connected graph $H$ be freely attachable to $\mathcal{A}$. Then there exists $\alpha>0$ such that

$$
\operatorname{Pr}\left[f_{H}\left(R_{n}\right) \leqslant \alpha n\right]=e^{-\Omega(n)} .
$$

This result shows for example that, if the $k$-leaf star rooted at its centre is freely attachable to $\mathcal{A}$, then $R_{n}$ has linearly many vertices of degree $k+1$, with exponentially small failure probability. It is possible to extend the theorem to consider graphs $H$ with (slowly) growing size, see for example Theorem 3.1 in [51], but we do not pursue that here. If $(\mathcal{A}, \tau)$ is well-behaved then we can be more precise about $f_{H}\left(R_{n}\right)$, extending Proposition 1.9 of [48].

Proposition 2.6. Let the weighted graph class $(\mathcal{A}, \tau)$ be well-behaved with growth constant $\gamma$, and let $R_{n} \in_{\tau} \mathcal{A}$. Let the connected graph $H$ be freely attachable to $\mathcal{A}$. Then

$$
\frac{f_{H}\left(R_{n}\right)}{n} \rightarrow \lambda \cdot \frac{\lambda^{e(H)}}{\gamma^{v(H)} v(H) !} \quad \text { in probability as } n \rightarrow \infty \text {. }
$$

The same result holds if we count disjoint pendant appearances. Indeed, if $\tilde{f}_{H}\left(R_{n}\right)$ denotes the number of pendant appearances of $H$ in $R_{n}$ that share a vertex or the root edge with some other pendant appearance of $H$, then $\mathbb{E}\left[\tilde{f}_{H}\left(R_{n}\right)\right]=O(1)$.

\subsection{Definition of a 'well-behaved' graph class}

Now at last in this section we can say precisely what we mean by a well-behaved class. We need first to introduce the notions of a graph class with a 'freely-addable-or-limited' dichotomy, and of a graph class 'maintaining at least factorial growth'. Along the way we introduce 'very well-behaved' graph classes.

\subsubsection{Freely-addable-or-limited classes of graphs}

Recall that, given a class $\mathcal{A}$ of graphs, the graph $H \in \mathcal{A}$ is freely addable to $\mathcal{A}$ if the disjoint union $G \cup H \in \mathcal{A}$ whenever $G \in \mathcal{A}$. We denote the class of graphs which are freely addable to $\mathcal{A}$ by $\mathcal{F}_{\mathcal{A}}$. For example, if $\mathcal{A}$ is the class $\mathcal{G}^{S}$ of graphs embeddable on a fixed surface $S$ then $\mathcal{F}_{\mathcal{A}}$ is the class $\mathcal{P}$ of planar graphs. Observe that if $\mathcal{A}$ is minor-closed and bridge-addable then $\mathcal{F}_{\mathcal{A}}$ is minor-closed and addable.

We say that a graph $H \in \mathcal{A}$ is limited in $\mathcal{A}$ if $k H$ is not in $\mathcal{A}$ for some positive integer $k$. Here $k H$ denotes the disjoint union of $k$ copies of $H$. For example, if $\mathcal{A}$ is $\mathcal{G}^{S}$ as above then the graphs in $\mathcal{A}$ which are limited in $\mathcal{A}$ are the non-planar graphs, which are exactly the non-freely addable graphs. If $\mathcal{A}$ is decomposable then no graph in $\mathcal{A}$ is limited in $\mathcal{A}$. Indeed if $H$ is freely addable to a class $\mathcal{A}$ then $H$ is not limited in $\mathcal{A}$ (recall that each graph in a decomposable class is freely addable to the class). 
We are interested in classes $\mathcal{A}$ of graphs such that each graph $G \in \mathcal{A}$ is either freely addable or limited (as we have noted it cannot be both): let us call such a graph class freely-addable-or-limited. From what we have just seen, for any surface $S$ the class $\mathcal{G}^{S}$ is freely-addable-or-limited. If $\mathcal{A}$ is decomposable then $\mathcal{F}_{\mathcal{A}}=\mathcal{A}$ so $\mathcal{A}$ is freely-addableor-limited. If $\mathcal{A}$ is the class $\operatorname{Ex}\left(k C_{3}\right)$ of graphs with at most $k$ vertex-disjoint cycles, then $\mathcal{F}_{\mathcal{A}}$ is the class of forests and each graph with a cycle is limited, so $\mathcal{A}$ is freelyaddable-or-limited. An example of a class which is not freely-addable-or-limited is the class $\operatorname{Ex}\left(C_{3} \cup C_{4}\right)$ of graphs with no minor $C_{3} \cup C_{4}$, where $C_{3}$ is neither freely-addable nor limited.

\subsubsection{Very well-behaved classes of graphs}

It is conjectured [6] that any proper minor-closed class $\mathcal{A}$ of graphs has a growth constant, and it is natural to conjecture similarly that $(\mathcal{A}, \tau)$ always has a growth constant. Part of the definition of $(\mathcal{A}, \tau)$ being well-behaved will require that there is a growth constant.

We (temporarily) call the weighted class $(\mathcal{A}, \tau)$ of graphs very well-behaved if it is minor-closed, bridge-addable and freely-addable-or-limited; and if it either is decomposable, or it is closed under subdividing edges and has a growth constant.

From what we have already seen, to show that $(\mathcal{A}, \tau)$ must be very well-behaved when $\mathcal{A}$ is a proper minor-closed addable class, or a class $\mathcal{G}^{S}$, it suffices to show that the growth constant must exist. This is done in Sections 3.2 and 3.3.

Now let $\mathcal{A}$ be the class of graphs with at most $k$ vertex-disjoint cycles. Then $\mathcal{A}$ is minor-closed, bridge-addable, freely-addable-or-limited and closed under subdividing edges. In the uniform case this class has growth constant $2^{k} e$ [43], and so it is very wellbehaved. Furthermore, straightforward adaptations of the proof in [43] shows that the weighted class $(\mathcal{A}, \tau)$ has a growth constant, and so it is very well-behaved. However, consider for example the class of graphs with no two vertex-disjoint cycles of length at least 4: this class is not decomposable nor closed under subdividing edges, and so it is not very well behaved. To cover such further graph classes, we weaken the condition and define a larger class of 'well-behaved' graph classes. Unfortunately the definition is more involved.

\subsubsection{Maintaining at least factorial growth}

We shall want to consider classes of graphs which we can show do not have any sudden dips in their growth rate. Let us say that a weighted graph class $(\mathcal{A}, \tau)$ of graphs maintains at least factorial growth if there exist an $\eta>0$ and a function $g(n)=(1+o(1))^{n}$ such that for each $n$ and each $j$ with $1 \leqslant j<n$ we have

$$
\tau\left(\mathcal{A}_{n}\right) \geqslant \tau\left(\mathcal{A}_{n-j}\right)(n)_{j} \eta^{j} g(n) .
$$

An equivalent condition avoiding the function $g$ is that there exist an $\eta>0$ such that for each $\epsilon>0$, for each sufficiently large $n$, for each $1 \leqslant j<n$

$$
\tau\left(\mathcal{A}_{n}\right) \geqslant \tau\left(\mathcal{A}_{n-j}\right)(n)_{j} \eta^{j} e^{-\epsilon n} .
$$

THE ELECTRONiC JOURNAL OF COMBINATORICS 20(2) (2013), \#P52 
It follows easily from lemma 3.1 (a) below that, if $\mathcal{A}$ is bridge-addable and $\mathcal{C}$ is the class of connected graphs in $\mathcal{A}$, then $(\mathcal{A}, \tau)$ maintains at least factorial growth if and only if $(\mathcal{C}, \tau)$ does.

These equivalent conditions are weaker than having a growth constant. Several weighted graph classes may be shown easily to maintain at least factorial growth, for example if $(\mathcal{A}, \tau)$ has a growth constant or if $\mathcal{A}$ is closed under subdividing edges - see Section 3.5 below. Since $(n)_{j} \geqslant(n / e)^{j}$ for $j=1, \ldots, n$, it would make no difference if we replaced $(n)_{j} \eta^{j}$ in the definition by $(\eta n)^{j}$. Also, to prove that $(\mathcal{A}, \tau)$ maintains at least factorial growth, by Lemma 3.6 it suffices to show that there is a $\delta>0$ such that (11) or (12) holds for each $j$ with $1 \leqslant j \leqslant \delta n$.

\subsubsection{Well-behaved classes of graphs}

We may at last say exactly what 'well-behaved' means. We start with the definition of a very well-behaved graph class $\mathcal{A}$, and we simply replace the condition that $\mathcal{A}$ be either decomposable or closed under subdividing edges by the condition that the subclass $\mathcal{A}^{\delta \geqslant 2}$ is either 'as small as the paths' or it is 'consistently large'. (Recall that $\mathcal{A}^{\delta \geqslant 2}$ is the class of graphs in $\mathcal{A}$ with minimum degree at least 2.) It will be easy to check that any very well-behaved graph class is well-behaved.

Definition The weighted class $(\mathcal{A}, \tau)$ of graphs is well-behaved if it is minor-closed, bridgeaddable, freely-addable-or-limited, and has a growth constant; and if the weighted class $\left(\mathcal{A}^{\delta \geqslant 2}, \tau\right)$ either (a) is empty or has radius of convergence at least $\lambda^{-1}$ or (b) maintains at least factorial growth.

From our observations in Section 2.1, we obtain an equivalent condition if we replace the assumption that $(\mathcal{A}, \tau)$ has a growth constant by the assumption that $(\mathcal{C}, \tau)$ has a growth constant, where $\mathcal{C}$ is the class of connected graphs in $\mathcal{A}$. Similarly, we could replace $\mathcal{A}^{\delta \geqslant 2}$ by the class of connected graphs in $\mathcal{A}$ with minimum degree at least 2 .

Lemma 2.7. Every very well-behaved weighted graph class is well-behaved, and in particular the weighted class $(\mathcal{A}, \tau)$ is well-behaved in the following cases, with any $\tau$ :

(a) $\mathcal{A}$ is minor-closed and addable,

(b) $\mathcal{A}$ is the class $\mathcal{G}^{S}$ of graphs embeddable on any given surface $S$,

(c) $\mathcal{A}$ is the class of graphs which contain at most $k$ vertex-disjoint cycles, for any given positive integer $k$.

\subsection{Plan of the rest of the paper}

The next section collects and proves various preliminary general results, and contains a proof of Lemma 2.7 above which shows that certain graph classes are well-behaved. After that, in Section 4, we prove the results stated in Section 2.1 on the Boltzmann Poisson random graph. The next section proves most of the results presented in Section 2.3 on smoothness and the 2-core; and the smoothness results allow us, after a brief section on 
Poisson convergence, to prove the results on $\operatorname{Frag}\left(R_{n}\right)$ and connectedness in Section 2.2. After that we prove the results on appearances given in Section 2.4, and finally we make some concluding remarks.

\section{Preliminary general results}

This section presents various preliminary general results, and gives a proof of Lemma 2.7, which shows that certain interesting graph classes are well-behaved.

\subsection{Connectivity bounds for a bridge-addable class}

We start with a lemma taken from [50], which will be used several times in this paper. Part (a) is a special case of Theorem 2.1 in [50], and extends Theorem 2.2 of [52]: part (b) is a special case of Theorem 2.2 in [50], and extends Lemmas 2.5 and 2.6 of [48]. (The paper [50] also gives asymptotic versions of these results, in the case when the class is closed also under deleting bridges, which match the results for forests described in 2.2.)

Lemma 3.1. Let the finite non-empty weighted set $(\mathcal{A}, \tau)$ of graphs be bridge-addable, and let $R \in_{\tau} \mathcal{A}$. Then

(a) $\kappa(R)$ is stochastically at most $1+\operatorname{Po}(\nu / \lambda)$, and so in particular

$\mathbb{P}(R$ is connected $) \geqslant e^{-\nu / \lambda} ;$ and

(b) $\mathbb{E}[\operatorname{frag}(R)]<2 \nu / \lambda$.

\subsection{Growth constant for an addable class}

The following result is an extension of Proposition 1.1 in [48], and its proof follows similar lines.

Lemma 3.2. Let $\mathcal{A}$ be a non-empty addable subclass of a proper minor-closed class of graphs, and consider any weighting $\tau$. Then there is a constant $\gamma$ with $0<\gamma<\infty$, which does not depend of $\nu$, such that

$$
\left(\tau\left(\mathcal{A}_{n}\right) / n !\right)^{1 / n} \rightarrow \gamma \text { as } n \rightarrow \infty
$$

Proof. Let $\mathcal{C}$ be the class of connected graphs in $\mathcal{A}$. Then $\tau\left(\mathcal{C}_{n}\right) \geqslant \tau\left(\mathcal{A}_{n}\right) e^{-\nu / \lambda}$ by Lemma 3.1 (a), and so for all positive integers $a$ and $b$

$$
\begin{aligned}
\tau\left(\mathcal{A}_{a+b}\right) & \geqslant \frac{1}{2}\left(\begin{array}{c}
a+b \\
a
\end{array}\right) \tau\left(\mathcal{C}_{a}\right) \tau\left(\mathcal{C}_{b}\right) \\
& \geqslant 2(a+b) ! \frac{\tau\left(\mathcal{A}_{a}\right) e^{-\nu / \lambda}}{2 a !} \frac{\tau\left(\mathcal{A}_{b}\right) e^{-\nu / \lambda}}{2 b !} .
\end{aligned}
$$

Thus, if we set $f(n)=\frac{\tau\left(\mathcal{A}_{n}\right) e^{-2 \nu / \lambda}}{2 n !}$ then $f(a+b) \geqslant f(a) f(b)$, that is $f$ is supermultiplicative.

Since $\mathcal{A}$ is a subclass of a proper minor-closed class of graphs, there is a constant $c_{1}$ such that $\left|\mathcal{A}_{n}\right| \leqslant n ! c_{1}^{n}$, see [55] (or [27] for a different proof). Also there is a constant $c_{2}$ 
such that each graph in $\mathcal{A}$ has average degree at most $c_{2}$, by a result of Mader [46] (see also for example Diestel [19]). Hence

$$
\tau\left(\mathcal{A}_{n}\right) \leqslant\left|\mathcal{A}_{n}\right| \max \left\{1, \lambda^{n-1}\right\} \max \left\{1, \lambda_{1}^{c_{2} n / 2}\right\} \max \left\{\nu, \nu^{n}\right\}
$$

Thus $\gamma=\sup _{n} f(n)^{1 / n}$ satisfies $0<\gamma<\infty$; and since $f$ is supermultiplicative it follows by Fekete's lemma (see for example [45] Lemma 11.6) that as $n \rightarrow \infty$ we have $f(n)^{1 / n} \rightarrow \gamma$ and so also $\left(\tau\left(\mathcal{A}_{n}\right) / n !\right)^{1 / n} \rightarrow \gamma$. Finally note that $\gamma$ cannot depend on $\nu$ since $\mathcal{A}$ contains all components (see the discussion following (3)).

\subsection{Growth constant for $\left(\mathcal{G}^{S}, \tau\right)$}

Since the class $\mathcal{P}$ of planar graphs is minor-closed and addable, we know from the last subsection that, with any weighting $\tau$, the weighted graph class $(\mathcal{P}, \tau)$ has a growth constant $\gamma(\mathcal{P}, \tau)$, which does not depend on $\nu$. We may show that $\left(\mathcal{G}^{S}, \tau\right)$ has the same growth constant $\gamma(\mathcal{P}, \tau)$, by induction on the Euler genus of $S$, following the treatment of the uniform case in [47].

\subsection{Freely-addable-or-limited graph classes}

It was shown in [47] that, for $R_{n} \in_{u} \mathcal{G}^{S}$ (the uniform case), the probability that $\operatorname{Frag}\left(R_{n}\right)$ is non-planar is $O(\ln n / n)$ : here we improve and extend this result. Let us write $G \succeq_{m} H$ to mean that $G$ has a minor (isomorphic to) $H$. First we give a lemma concerning a single unwanted minor.

Lemma 3.3. Let $\mathcal{A}$ be bridge-addable, let $R_{n} \in_{\tau} \mathcal{A}$, let $H$ be a connected graph, and let $k$ be a non-negative integer. Then

$$
\mathbb{P}\left(\operatorname{Frag}\left(R_{n}\right) \succeq_{m} H\right) \leqslant \frac{3 \nu k}{2 \lambda} \frac{\ln n}{n}+\frac{6 \nu}{\lambda n}+\mathbb{P}\left(R_{n} \succeq_{m}(k+1) H\right) .
$$

Further if $H$ is 2-connected then we may improve the first term in the bound to $\frac{3 \nu k}{2 \lambda v(H)} \frac{1}{n}$.

Proof. Let $\omega=\omega(n)=\lfloor n / 3\rfloor$. Let $\mathcal{B}_{n}^{j}$ be the class of graphs $G \in \mathcal{A}_{n}$ such that $G \in$ $\operatorname{Ex}(k+1) H, \operatorname{frag}(G) \leqslant \omega, \operatorname{Frag}(G) \succeq_{m} H$, and there are exactly $j$ vertices in the lex-first component $C$ of $\operatorname{Frag}(G)$ with minor $H$. Given a graph $G \in \mathcal{B}_{n}^{j}$, add any edge between this component $C$ and a vertex in $\operatorname{Big}(G)$, to form $G^{\prime}$. This gives $j \cdot \operatorname{big}(G) \geqslant j \cdot 2 n / 3$ constructions of graphs $G^{\prime} \in \mathcal{A}$.

Each graph $G^{\prime}$ constructed can have at most $k$ (oriented) bridges $u v$ such that the component of $G^{\prime}-u v$ containing $u$ has order at least $2 n / 3$, and the component containing $v$ has order $j$ and has a minor $H$ (since otherwise the original graph $G$ is not in $\operatorname{Ex}(k+1) H$. Thus $G^{\prime}$ can be constructed at most $k$ times. Hence

$$
j \frac{2 n}{3} \frac{\lambda}{\nu} \tau\left(\mathcal{B}_{n}^{j}\right) \leqslant k \tau\left(\mathcal{A}_{n}\right)
$$


SO

$$
\tau\left(\mathcal{B}_{n}^{j}\right) \leqslant \frac{1}{j} \frac{3 \nu k}{2 \lambda n} \tau\left(\mathcal{A}_{n}\right)
$$

Let $\mathcal{B}_{n}=\cup_{j \leqslant \omega} \mathcal{B}_{n}^{j}$. Since $\sum_{v(H) \leqslant j \leqslant \omega} 1 / j \leqslant \ln \omega$ we have

$$
\tau\left(\mathcal{B}_{n}\right) \leqslant \frac{3 \nu k}{2 \lambda} \frac{\ln n}{n} \tau\left(\mathcal{A}_{n}\right)
$$

Thus $\tau\left(\left\{G \in \mathcal{A}_{n}: \operatorname{Frag}(G) \succeq_{m} H\right\}\right)$ is at most

$$
\frac{3 \nu k}{2 \lambda} \frac{\ln n}{n} \tau\left(\mathcal{A}_{n}\right)+\tau\left(\left\{G \in \mathcal{A}_{n}: \operatorname{frag}(G)>\omega\right\}\right)+\tau\left(\left\{G \in \mathcal{A}_{n}: G \succeq_{m}(k+1) H\right\}\right) .
$$

But recall from Lemma 3.1 (b) that $\mathbb{E}\left[\operatorname{frag}\left(R_{n}\right)\right]<2 \nu / \lambda$, and so

$$
\mathbb{P}\left(\operatorname{frag}\left(R_{n}\right)>n / 3\right)<\frac{6 \nu}{\lambda n} .
$$

Hence we may divide by $\tau\left(\mathcal{A}_{n}\right)$ in the last displayed inequality to obtain (13).

Now suppose that $H$ is 2 -connected. Let $G \in \mathcal{B}_{n}$, where $\mathcal{B}$ is as above. Then some block of some component of $\operatorname{Frag}(G)$ has a minor $H$. Add any edge between a vertex in such a block and a vertex in $\operatorname{Big}(G)$, to form $G^{\prime}$. This gives at least $v(H) \cdot \operatorname{big}(G) \geqslant 2 v(H) n / 3$ constructions of graphs $G^{\prime} \in \mathcal{A}$.

Each graph $G^{\prime}$ constructed can have at most $k$ (oriented) bridges $u v$ such that the component of $G^{\prime}-u v$ containing $u$ has order at least $2 n / 3$, and the component containing $v$ is in a block with a minor $H$. Thus $G^{\prime}$ can be constructed at most $k$ times. Hence

$$
v(H) \frac{2 n}{3} \frac{\lambda}{\nu} \tau\left(\tilde{\mathcal{B}}_{n}\right) \leqslant k \tau\left(\mathcal{A}_{n}\right)
$$

and we may proceed as before.

Recall that $\mathcal{F}_{\mathcal{A}}$ denotes the class of graphs which are freely addable to $\mathcal{A}$. The last lemma yields:

Lemma 3.4. Let $\mathcal{A}$ be minor-closed, bridge-addable and freely-addable-or-limited; and let $R_{n} \in_{\tau} \mathcal{A}$ for $n=1,2, \ldots$ Then there is a constant $c$ such that

$$
\mathbb{P}\left(\operatorname{Frag}\left(R_{n}\right) \notin \mathcal{F}_{\mathcal{A}}\right) \leqslant \frac{c \nu}{n \lambda} \quad \text { for each } n \text {. }
$$

Proof. As we noted in Section 2.5.1, $\mathcal{F}_{\mathcal{A}}$ is minor-closed and addable, with a finite set of excluded minors each of which is 2-connected. Thus it suffices to prove that for each excluded minor $K$ for $\mathcal{F}_{\mathcal{A}}$ there is a constant $c_{K}$ such that $\mathbb{P}\left(\operatorname{Frag}\left(R_{n}\right) \succeq_{m} K\right) \leqslant \frac{c_{K} \nu}{n \lambda}$ for each $n$. But if $K \in \mathcal{A}$ then $K$ must be limited in $\mathcal{A}$ since $\mathcal{A}$ is freely-addable-or-limited, so there is a $k$ such that $(k+1) K \notin \mathcal{A}$; and now we may use Lemma 3.3 for the 2-connected graph $K$. 


\subsection{Maintaining at least factorial growth}

We shall want to consider weighted graph classes $(\mathcal{A}, \tau)$ (with each vertex degree at least 2) which maintain at least factorial growth, as defined in Section 2.5.3.

It is easy to see that if $(\mathcal{A}, \tau)$ has growth constant $\gamma>0$ then $(\mathcal{A}, \tau)$ maintains at least factorial growth. For let $0<\epsilon<1$ (for example take $\epsilon=\frac{1}{2}$ ) and let $n_{0}$ be such that $(1-\epsilon)^{n} n ! \gamma^{n} \leqslant \tau\left(\mathcal{A}_{n}\right) \leqslant(1-\epsilon)^{-n} n ! \gamma^{n}$ for each $n \geqslant n_{0}$. Then for each $n>n_{0}$ and $1 \leqslant j \leqslant n-n_{0}$,

$$
\tau\left(\mathcal{A}_{n}\right) \geqslant(1-\epsilon)^{n} n ! \gamma^{n}=(1-\epsilon)^{n}(n-j) ! \gamma^{n-j}(n)_{j} \gamma^{j} \geqslant(1-\epsilon)^{2 n} \tau\left(\mathcal{A}_{n-j}\right)(n)_{j} \gamma^{j},
$$

and (11) follows for each $1 \leqslant j<n$.

Other graph classes may be shown to maintain at least factorial growth even though we may not know whether they have a growth constant, and indeed that is the point of introducing this property. We shall see below that this holds for example when the class is closed under subdividing edges. Note in particular that for any given surface $S$, the class of graphs $G$ which have minimum degree at least 2 and are embeddable on $S$ is closed under subdividing edges.

Given a class $\mathcal{A}$ of graphs and a graph $G \in \mathcal{A}$, let us say that an edge $e$ in $G$ is arbitrarily subdividable if $\mathcal{A}$ contains each graph obtained by repeatedly subdividing $e$.

Lemma 3.5. Let $\mathcal{A}$ be a non-empty class of graphs, and suppose that each graph in $\mathcal{A}$ contains an arbitrarily subdividable edge. Then for each $\tau$, the weighted class $(\mathcal{A}, \tau)$ maintains at least factorial growth.

Proof. $\quad$ Let $1 \leqslant j<n$ be such that $\mathcal{A}_{n-j}$ is non-empty. Pick a set $S$ of $j$ vertices from $[n]$, and list them as $v_{1}, v_{2}, \ldots, v_{j}$. Pick a graph $G$ in $\mathcal{A}$ on $[n] \backslash S$ and an arbitrarily subdividable edge $e=u w$ where $u<w$, and replace $e$ by the path $u v_{1} v_{2} \cdots v_{j} w$, to form $G^{\prime}$. The weighted number of constructions is at least $\tau\left(\mathcal{A}_{n-j}\right)(n)_{j} \eta^{j}$, where $\eta=\lambda$.

How often can a given graph $G^{\prime}$ on $[n]$ be constructed? In $G^{\prime}$ there must be a path $v_{1}, v_{2}, \ldots, v_{j}$ of vertices of degree 2 , where $j$ is known. The number of such paths is at most $n^{2}$. But knowing the path determines $G, e$ and the list, so $G^{\prime}$ can be constructed at most $n^{2}$ times. Hence

$$
\tau\left(\mathcal{A}_{n}\right) \geqslant \tau\left(\mathcal{A}_{n-j}\right)(n){ }_{j} \eta^{j} g(n),
$$

where $g(n)=n^{-2}$, and the lemma follows.

Finally here let us check that a seemingly more 'local' and weaker condition implies that $\mathcal{A}$ maintains at least factorial growth (as defined at (12)).

Lemma 3.6. Assume that $\mathcal{A}_{n} \neq \emptyset$ for infinitely many $n$. Let $0<\delta \leqslant 1,0<\eta \leqslant 1$ and $g(n)=(1+o(1))^{n}$; and suppose that

$$
\tau\left(\mathcal{A}_{n}\right) \geqslant \tau\left(\mathcal{A}_{n-j}\right)(n)_{j} \eta^{j} g(n)
$$

for each sufficiently large $n$ and each $1 \leqslant j \leqslant \delta n$. Then $\mathcal{A}$ maintains at least factorial growth (with the same $\eta$ ). 
Proof. Let $0<\epsilon \leqslant 1$. Let $N_{0}$ be sufficiently large that $g(n) \geqslant(1-\delta \epsilon / 2)^{n}$ for all $n \geqslant N_{0}$. Let $N \geqslant N_{0}$ be such that $\mathcal{A}_{N} \neq \emptyset$. Let us first show that

$$
\tau\left(\mathcal{A}_{n}\right) \geqslant(n)_{j} \eta^{j} e^{-\epsilon n} \cdot \tau\left(\mathcal{A}_{n-j}\right)
$$

for each $n>N$ and $j=1, \ldots, n-N$.

Let $n>N$ and $1 \leqslant j \leqslant n-N$. Let $n_{i}=\left\lfloor(1-\delta)^{i} n\right\rfloor$ for $i=0,1,2, \ldots$. Let $i_{0}$ be the least $i$ such that $n_{i} \leqslant n-j$, and redefine $n_{i_{0}}$ as $n-j$. Observe that $\sum_{i=0}^{i_{0}-1} n_{i} \leqslant n \sum_{i \geqslant 0}(1-\delta)^{i}=$ $n / \delta$. Note also that $(1-x / 2) \geqslant e^{-x}$ for $0<x \leqslant 1$ and so $(1-\delta \epsilon / 2)^{1 / \delta} \geqslant e^{-\epsilon}$. Hence

$$
\prod_{i=0}^{i_{0}-1} g\left(n_{i}\right) \geqslant(1-\delta \epsilon / 2)^{\sum_{i=0}^{i_{0}-1} n_{i}} \geqslant(1-\delta \epsilon / 2)^{n / \delta} \geqslant e^{-\epsilon n} .
$$

Now

$$
\begin{aligned}
\tau\left(\mathcal{A}_{n}\right) & \geqslant \prod_{i=0}^{i_{0}-1}\left(n_{i}\right)_{\left(n_{i}-n_{i+1}\right)} \eta^{n_{i}-n_{i+1}} g\left(n_{i}\right) \cdot \tau\left(\mathcal{A}_{n_{i_{0}}}\right) \\
& =(n)_{j} \eta^{j} \cdot \prod_{i=0}^{i_{0}-1} g\left(n_{i}\right) \cdot \tau\left(\mathcal{A}_{n-j}\right) \\
& \geqslant(n)_{j} \eta^{j} e^{-\epsilon n} \cdot \tau\left(\mathcal{A}_{n-j}\right)
\end{aligned}
$$

as required, and we have proved (14).

Let $\alpha=\max _{1 \leqslant i \leqslant N}\left\{\tau\left(\mathcal{A}_{i}\right) / \tau\left(\mathcal{A}_{N}\right)\right\}$. Let $c=(\alpha N !)^{-1}$, and note that $0<c \leqslant 1$. We want to establish (12). It will suffice to show that for each $n>N$,

$$
\tau\left(\mathcal{A}_{n}\right) \geqslant \tau\left(\mathcal{A}_{n-j}\right)(n)_{j} \eta^{j} e^{-\epsilon n} \cdot c
$$

for each $n>N$ and $j=1, \ldots, n-1$. Since $c \leqslant 1$ this is immediate from (14) for $j \leqslant n-N$. So assume that $n-N<j<n$, and let $k=j-(n-N)$ so $1 \leqslant k \leqslant N-1$. By (14) with $j=n-N$,

$$
\tau\left(\mathcal{A}_{n}\right) \geqslant \tau\left(\mathcal{A}_{N}\right)(n)_{n-N} \eta^{n-N} e^{-\epsilon n}
$$

But

$$
\tau\left(\mathcal{A}_{N}\right) \geqslant \tau\left(\mathcal{A}_{N-k}\right) \cdot \alpha^{-1} \geqslant \tau\left(\mathcal{A}_{N-k}\right)(N)_{k} \eta^{k} \cdot c .
$$

Putting the last two inequalities together gives

$$
\tau\left(\mathcal{A}_{n}\right) \geqslant \tau\left(\mathcal{A}_{n-j}\right)(n)_{j} \eta^{j} e^{-\epsilon n} \cdot c
$$

as required. 


\subsection{Being well-behaved: proof of Lemma 2.7}

If $\mathcal{A}$ is an addable proper minor-closed class of graphs then $\mathcal{A}$ is decomposable, and $(\mathcal{A}, \tau)$ has a growth constant by lemma 3.2 ; and if $\mathcal{A}$ is $\mathcal{G}^{S}$, or $\mathcal{A}$ is the class of graphs which contain at most $k$ vertex-disjoint cycles, then $\mathcal{A}$ is closed under subdividing edges, and $(\mathcal{A}, \tau)$ has a growth constant. Also in each case $\mathcal{A}$ is freely-addable-or-limited, as we saw in Section 2.5.1, and so $(\mathcal{A}, \tau)$ is very well-behaved.

It remains to show that each very well-behaved weighted graph class $(\mathcal{A}, \tau)$ is wellbehaved. If $\mathcal{A}^{\delta \geqslant 2}$ is empty then $(\mathcal{A}, \tau)$ is well-behaved, so we may assume that $\mathcal{A}^{\delta \geqslant 2}$ is non-empty.

Suppose first that $\mathcal{A}$ is closed under subdividing edges. Then also $\mathcal{A}^{\delta \geqslant 2}$ is closed under subdividing edges, so by Lemma $3.5\left(\mathcal{A}^{\delta \geqslant 2}, \tau\right)$ maintains at least factorial growth; and so $(\mathcal{A}, \tau)$ is well-behaved.

So suppose now that $\mathcal{A}$ is decomposable. Then $\mathcal{A}$ is addable, and so also $\mathcal{A}^{\delta \geqslant 2}$ is addable. By Lemma 3.2, $\left(\mathcal{A}^{\delta \geqslant 2}, \tau\right)$ has a growth constant, and so a fortiori it maintains at least factorial growth, as noted in Section 2.5.3. This completes the proof.

\section{7 $\rho(\mathcal{A}, \tau)$ as $\tau$ varies}

Consider a proper minor-closed class $\mathcal{A}$ of graphs, with $\rho(\mathcal{A},(1,1))$ finite. For example if $\mathcal{A}$ contains the class $\mathcal{F}$ of forests then $\rho(\mathcal{A},(1,1)) \leqslant \rho(\mathcal{F},(1,1))=e^{-1}<\infty$. We saw that the radius of convergence $\rho(\mathcal{A}, \tau)$ does not depend on the component parameter $\nu$. Let us write $\rho(\lambda)$ for $\rho(\mathcal{A}, \tau)$.

Let $c=c(\mathcal{A})$ be the maximum average degree of a graph in $\mathcal{A}$, and recall that $c$ is finite (see the proof of Lemma 3.2). Note that $c \geqslant 2$ : for if $c<2$ and $\mathcal{B}$ is a class of all graphs with average degree at most $c$, then

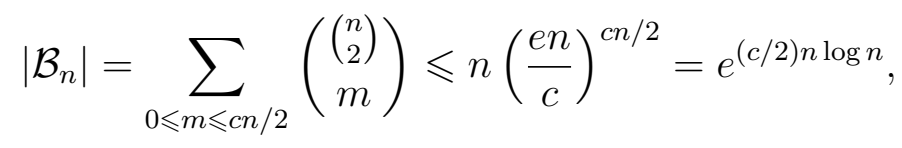

so $\left(\left|\mathcal{B}_{n}\right| / n !\right)^{1 / n}=o(1)$, that is $\rho(\mathcal{B}, \lambda)=0$. (See [33] for related details.)

Proposition 3.7. The function $\rho(\mathcal{A}, \lambda)$ is continuous and strictly decreasing on $(0, \infty)$. If $0<\lambda \leqslant 1$ then

$$
\rho(\mathcal{A}, 1) \lambda^{-1} \leqslant \rho(\mathcal{A}, \lambda) \leqslant \rho(\mathcal{A}, 1) \lambda^{-c / 2},
$$

and if $\lambda \geqslant 1$ then

$$
\rho(\mathcal{A}, 1) \lambda^{-c / 2} \leqslant \rho(\mathcal{A}, \lambda) \leqslant \rho(\mathcal{A}, 1) \lambda^{-1} .
$$

For the class $\mathcal{F}$ of forests we have $c=2$, and

$$
\rho(\mathcal{F}, \lambda)=\rho(\mathcal{F}, \mathbf{1}) \lambda^{-1}=(e \lambda)^{-1}
$$

as we already noted; and we see that the inequalites above for $\rho(\mathcal{A}, \lambda)$ are tight. 
Proof. To show $\rho(\mathcal{A}, 1) \lambda^{-1} \leqslant \rho(\mathcal{A}, \lambda)$ for $0<\lambda \leqslant 1$. Let $0<\epsilon<1$. Let $\mathcal{A}^{\prime}=\{G \in \mathcal{A}$ : $e(G) \leqslant(1-\epsilon) v(G)\}$, and let $\mathcal{A}^{\prime \prime}=\mathcal{A} \backslash \mathcal{A}^{\prime}$. Then $\rho\left(\mathcal{A}^{\prime}, \lambda\right)=\infty$ as we saw above, and $\rho\left(\mathcal{A}^{\prime \prime}, \lambda\right)=\rho(\mathcal{A}, \lambda)$. Then for $\lambda \leqslant 1$

$$
\tau\left(\mathcal{A}_{n}^{\prime \prime}\right) \leqslant\left|\mathcal{A}_{n}^{\prime \prime}\right| \lambda^{(1-\epsilon) n} \leqslant\left|\mathcal{A}_{n}\right| \lambda^{(1-\epsilon) n},
$$

and so $\rho(\mathcal{A}, \lambda)=\rho\left(\mathcal{A}^{\prime \prime}, \lambda\right) \geqslant \rho(\mathcal{A}, \mathbf{1}) \lambda^{-(1-\epsilon)}$. But this holds for each $0<\epsilon<1$ so $\rho(\mathcal{A}, \lambda) \geqslant \rho(\mathcal{A}, \mathbf{1}) \hat{\lambda}^{-1}$. (We needed no assumptions on $\mathcal{A}$ here.)

To show $\rho(\mathcal{A}, \lambda) \leqslant \rho(\mathcal{A}, 1) \lambda^{-1}$ for $\lambda \geqslant 1$. As we saw following (3), $\rho(\mathcal{C}, \lambda)=\rho(\mathcal{A}, \lambda)$, where $\mathcal{C}$ is the class of connected graphs in $\mathcal{A}$. Thus for $\lambda \geqslant 1, \tau\left(\mathcal{C}_{n}\right) \geqslant\left|\mathcal{C}_{n}\right| \lambda^{n-1}$, so $\rho(\mathcal{C}, \lambda) \leqslant \rho(\mathcal{C}, \mathbf{1}) / \lambda$ and hence $\rho(\mathcal{A}, \lambda) \leqslant \rho(\mathcal{A}, \mathbf{1}) / \lambda$.

For the remaining inequalities, observe that $\tau\left(\mathcal{A}_{n}\right)=\sum_{G \in \mathcal{A}_{n}} \lambda^{e(G)}$. Thus if $\lambda \leqslant 1$, $\tau\left(\mathcal{A}_{n}\right) \geqslant\left|\mathcal{A}_{n}\right| \lambda^{c n / 2}$, and so $\rho(\mathcal{A}, \lambda) \leqslant \rho(\mathcal{A}, \mathbf{1}) \lambda^{-c / 2}$; and if $\lambda \geqslant 1, \tau\left(\mathcal{A}_{n}\right) \leqslant\left|\mathcal{A}_{n}\right| \lambda^{c n / 2}$, and so $\rho(\mathcal{A}, \lambda) \geqslant \rho(\mathcal{A}, \mathbf{1}) \lambda^{-c / 2}$.

To show that $\rho(\mathcal{A}, \lambda)$ is strictly decreasing on $(0, \infty)$, consider $\mathcal{A}^{\prime \prime}$ from the first part of the proof, with say $\epsilon=\frac{1}{2}$. Let $\eta>0$. Then

$$
\sum_{G \in \mathcal{A}_{n}^{\prime \prime}}((1+\eta) \lambda)^{e(G)} \geqslant(1+\eta)^{n / 2} \cdot \sum_{G \in \mathcal{A}_{n}^{\prime \prime}} \lambda^{e(G)}
$$

and so

$$
\rho(\mathcal{A},(1+\eta) \lambda)=\rho\left(\mathcal{A}^{\prime \prime},(1+\eta) \lambda\right) \leqslant(1+\eta)^{-\frac{1}{2}} \rho(\mathcal{A}, \lambda) .
$$

Now to show that $\rho(\mathcal{A}, \lambda)$ is continuous on $(0, \infty)$, observe that

$$
\sum_{G \in \mathcal{A}_{n}}((1+\eta) \lambda)^{e(G)} \leqslant(1+\eta)^{c n / 2} \cdot \sum_{G \in \mathcal{A}_{n}} \lambda^{e(G)}
$$

and so

$$
\rho(\mathcal{A},(1+\eta) \lambda) \geqslant(1+\eta)^{-c / 2} \rho(\mathcal{A}, \lambda)
$$

\section{Distribution of the Boltzmann random graph}

Let us first check equation (4). Recall that the class $\mathcal{A}$ of graphs is closed under isomorphism. We identify an unlabelled graph on $n$ vertices with an equivalence class under graph isomorphism of graphs on vertex set $[n]$. Since each graph $H \in \mathcal{U} \mathcal{A}_{n}$ consists of $\frac{n !}{\operatorname{aut}(H)} \operatorname{graphs} G \in \mathcal{A}_{n}$, we have

$$
\frac{x^{v(H)} y^{e(H)} z^{\kappa(H)}}{\operatorname{aut}(H)}=\sum_{G \in H} \frac{\operatorname{aut}(H)}{v(H) !} \frac{x^{v(H)} y^{e(H)} z^{\kappa(H)}}{\operatorname{aut}(H)}=\sum_{G \in H} \frac{x^{v(G)} y^{e(G)} z^{\kappa(G)}}{v(G) !} .
$$

Thus

$$
A(x, y, z)=\sum_{H \in \mathcal{U}_{\mathcal{A}}} \sum_{G \in H} \frac{x^{v(G)} y^{e(G)} z^{\kappa(G)}}{v(G) !}=\sum_{H \in \mathcal{U}_{\mathcal{A}}} \frac{x^{v(H)} y^{e(H)} z^{\kappa(H)}}{\operatorname{aut}(H)},
$$

proving (4). 
Proof of Theorem 2.1 Each sum and product below is over all $H$ in $\mathcal{U C}$. Let the unlabelled graph $G$ consist of $n_{H}$ components isomorphic to $H$ for each $H \in \mathcal{U C}$, where $0 \leqslant \sum_{H} n_{H}<\infty$. Then

$$
\rho^{v(G)}=\prod_{H} \rho^{v(H) n_{H}}, \quad \lambda^{e(G)}=\prod_{H} \lambda^{e(H) n_{H}}, \quad \nu^{\kappa(G)}=\prod_{H} \nu^{n_{H}}
$$

and

$$
\operatorname{aut}(G)=\prod_{H} \operatorname{aut}(H)^{n_{H}} n_{H} !
$$

Hence

$$
\frac{\rho^{v(G)} \lambda^{e(G)} \nu^{\kappa(G)}}{\operatorname{aut}(G)}=\prod_{H} \frac{\mu(H)^{n_{H}}}{n_{H} !} .
$$

Also since $\sum_{H} \mu(H)=C(\rho, \tau)$ by (4) applied to $\mathcal{C}$,

$$
\frac{1}{A(\rho, \tau)}=e^{-C(\rho, \tau)}=\prod_{H} e^{-\mu(H)} .
$$

Hence

$$
\begin{aligned}
\mathbb{P}[R=G] & =e^{-C(\rho, \tau)} \frac{\rho^{v(G)} \lambda^{e(G)} \nu^{\kappa(G)}}{\operatorname{aut}(G)} \\
& =\prod_{H} e^{-\mu(H)} \frac{\mu(H)^{n_{H}}}{n_{H} !} \\
& =\prod_{H} \mathbb{P}\left[\operatorname{Po}(\mu(H))=n_{H}\right] .
\end{aligned}
$$

Thus the probability factors appropriately, and the family of random variables $\kappa(R, H)$ for $H \in \mathcal{U C}$ satisfies

$$
\mathbb{P}\left[\kappa(R, H)=n_{H} \quad \forall H \in \mathcal{U C}\right]=\prod_{H} \mathbb{P}\left[\kappa(R, H)=n_{H}\right] .
$$

This holds for every choice of non-negative integers $n_{H}$ with $\sum_{H \in \mathcal{U C}} n_{H}<\infty$, and thus also without this last restriction (since both sides are zero if the sum is infinite). This completes the proof of the theorem.

\section{Smoothness and 2-core: proof of Theorem 2.4}

In this section we deduce Theorem 2.4, after two preliminary subsections: one on deducing smoothness for a class $\mathcal{A}$ from smoothness for the class of connected graphs in $\mathcal{A}$, and one on smoothness for classes of connected graphs. 


\subsection{Smoothness: from connected to general}

Lemma 5.1. Let the class $\mathcal{A}$ of graphs be bridge-addable, and let $R_{n} \in_{\tau} \mathcal{A}$. Let $\mathcal{F}$ denote the class $\mathcal{F}_{\mathcal{A}}$ of graphs freely addable to $\mathcal{A}$, and suppose that whp $\operatorname{Frag}\left(R_{n}\right) \in \mathcal{F}$. Let $\mathcal{C}$ be the class of connected graphs in $\mathcal{A}$, let $\rho=\rho(\mathcal{C}, \tau)$ satisfy $0<\rho<\infty$, and suppose further that $(\mathcal{C}, \tau)$ is smooth. Then $F(\rho, \tau)$ is finite, where $F$ is the exponential generating function for $\mathcal{F}$; and the weighted class $(\mathcal{A}, \tau)$ is smooth.

Proof of Lemma 5.1 Recall that $F(x, \tau)=\sum_{j \geqslant 0} \tau\left(\mathcal{F}_{j}\right) x^{j} / j$ !. We shall show that $F(\rho, \tau)$ is finite and $\tau\left(\mathcal{A}_{n}\right) \sim F(\rho, \tau) \tau\left(\mathcal{C}_{n}\right)$, from which it will follow immediately that $(\mathcal{A}, \tau)$ is smooth. Let $0<\eta<1$. Then we are to show that $F(\rho, \tau)$ is finite, and that for $n$ sufficiently large

$$
(1-\eta) F(\rho, \tau) \tau\left(\mathcal{C}_{n}\right) \leqslant \tau\left(\mathcal{A}_{n}\right) \leqslant(1+\eta) F(\rho, \tau) \tau\left(\mathcal{C}_{n}\right) .
$$

Let $\epsilon>0$ be sufficiently small that $\epsilon \leqslant 1 /\left(e^{\nu / \lambda}+2\right)$, and $(1-2 \epsilon)^{-1}(1+\epsilon) \leqslant 1+\eta$ and $(1-\epsilon)^{2} \geqslant 1-\eta$. By our assumptions and Lemma 3.1 (b), we may fix positive integers $k$ and $n_{0}$ sufficiently large that $\sum_{j=0}^{k} \tau\left(\mathcal{F}_{j}\right) \rho^{j} / j$ ! is at least $(1-\epsilon) F(\rho, \tau)$ if $F(\rho, \tau)$ is finite, and is at least $e^{\nu / \lambda}+2$ otherwise; and $\mathbb{P}\left[\operatorname{frag}\left(R_{n}\right)>k\right]<\epsilon$ and $\mathbb{P}\left[\operatorname{Frag}\left(R_{n}\right) \notin \mathcal{F}\right]<\epsilon$ for all $n \geqslant n_{0}$.

Since $(\mathcal{C}, \tau)$ is smooth, there exists $n_{1} \geqslant n_{0}$ sufficiently large that for all $n \geqslant n_{1}$, the ratio $\tilde{r}_{n}=n \tau\left(\mathcal{C}_{n-1}\right) / \tau\left(\mathcal{C}_{n}\right)$ satisfies

$$
(1-\epsilon)^{1 / k} \rho<\tilde{r}_{n}<(1+\epsilon)^{1 / k} \rho .
$$

Then for each $n \geqslant n_{1}+k$ and each $j=1, \ldots, k$, since

$$
\frac{(n)_{j} \tau\left(\mathcal{C}_{n-j}\right)}{\tau\left(\mathcal{C}_{n}\right)}=\prod_{i=1}^{j} \tilde{r}_{n-i+1}
$$

we have

$$
(1-\epsilon) \rho^{j}<\frac{(n)_{j} \tau\left(\mathcal{C}_{n-j}\right)}{\tau\left(\mathcal{C}_{n}\right)}<(1+\epsilon) \rho^{j} .
$$

Denote $\sum_{j=0}^{k}\left(\begin{array}{l}n \\ j\end{array}\right) \tau\left(\mathcal{F}_{j}\right) \tau\left(\mathcal{C}_{n-j}\right)$ by $\tilde{a}_{n}$. Observe that $\tilde{a}_{n} \leqslant \tau\left(\mathcal{A}_{n}\right)$ : we shall see that $\tilde{a}_{n}$ is an approximation to $\tau\left(\mathcal{A}_{n}\right)$. Note that

$$
\tilde{a}_{n}=\tau\left(\mathcal{C}_{n}\right) \sum_{j=0}^{k} \frac{\tau\left(\mathcal{F}_{j}\right)}{j !} \frac{(n)_{j} \tau\left(\mathcal{C}_{n-j}\right)}{\tau\left(\mathcal{C}_{n}\right)}
$$

and so for each $n \geqslant n_{1}+k$ we have

$$
(1-\epsilon) \tau\left(\mathcal{C}_{n}\right) \sum_{j=0}^{k} \frac{\tau\left(\mathcal{F}_{j}\right) \rho^{j}}{j !} \leqslant \tilde{a}_{n} \leqslant(1+\epsilon) \tau\left(\mathcal{C}_{n}\right) \sum_{j=0}^{k} \frac{\tau\left(\mathcal{F}_{j}\right) \rho^{j}}{j !} .
$$


We may now see that $F(\rho, \tau)$ is finite. For suppose not. Then for each $n \geqslant n_{1}+k$

$$
\tau\left(\mathcal{A}_{n}\right) \geqslant \tilde{a}_{n} \geqslant(1-\epsilon)\left(e^{\nu / \lambda}+2\right) \tau\left(\mathcal{C}_{n}\right) \geqslant\left(e^{\nu / \lambda}+1\right) \tau\left(\mathcal{C}_{n}\right)
$$

But since $\mathcal{A}$ is bridge-addable, by Lemma 3.1 the probability that $R_{n}$ is connected is at least $e^{-\nu / \lambda}$, and we obtain the contradiction that

$$
e^{\nu / \lambda} \cdot \tau\left(\mathcal{C}_{n}\right) \geqslant \tau\left(\mathcal{A}_{n}\right) \geqslant\left(e^{\nu / \lambda}+1\right) \cdot \tau\left(\mathcal{C}_{n}\right)
$$

Hence $F(\rho, \tau)$ must be finite.

From the above we have $\tilde{a}_{n} \leqslant(1+\epsilon) \tau\left(\mathcal{C}_{n}\right) F(\rho, \tau)$, and $\tilde{a}_{n} \geqslant(1-\epsilon)^{2} \tau\left(\mathcal{C}_{n}\right) F(\rho, \tau)$. But

$$
\tau\left(\mathcal{A}_{n}\right)=\tilde{a}_{n}+\sum_{G}\left\{\tau(G): G \in \mathcal{A}_{n}, \operatorname{frag}(G)>k \text { or } \operatorname{Frag}(G) \notin \mathcal{F}\right\} .
$$

Thus $\tau\left(\mathcal{A}_{n}\right) \leqslant \tilde{a}_{n}+2 \epsilon \tau\left(\mathcal{A}_{n}\right)$, and so

$$
\tau\left(\mathcal{A}_{n}\right) \leqslant(1-2 \epsilon)^{-1} \tilde{a}_{n} \leqslant(1+\eta) \tau\left(\mathcal{C}_{n}\right) F(\rho, \tau)
$$

and

$$
\tau\left(\mathcal{A}_{n}\right) \geqslant \tilde{a}_{n} \geqslant(1-\epsilon)^{2} \tau\left(\mathcal{C}_{n}\right) F(\rho, \tau) \geqslant(1-\eta) \tau\left(\mathcal{C}_{n}\right) F(\rho, \tau)
$$

So (16) holds and we are done.

We need Lemma 5.1 for graph classes which may not be decomposable (such as $\mathcal{G}^{S}$ ) but let us note here an elegant general result for a decomposable class $\mathcal{A}$ and the corresponding class $\mathcal{C}$ of connected graphs: by Corollary 4.3 of Bell and Burris [1], if $(\mathcal{C}, \tau)$ is smooth then so is $(\mathcal{A}, \tau)$.

\subsection{Connected graphs and smoothness}

Let us call a class $\mathcal{A}$ of graphs trimmable if it satisfies $G \in \mathcal{A} \Leftrightarrow 2$ - $\operatorname{core}(G) \in \mathcal{A}$. (To tell if a graph is in such a class, it does not matter if we repeatedly trim off leaves.) Recall that by convention the empty graph is in $\mathcal{A}$, so if $\mathcal{A}$ is trimmable then $\mathcal{A}$ contains every forest. Observe also that a minor-closed class of graphs is trimmable if and only if each excluded minor $H$ has minimum degree $\delta(H) \geqslant 2$. Also, if $\mathcal{A}$ is bridge-addable and monotone (that is, closed under forming subgraphs) then the class $\mathcal{C}$ of connected graphs in $\mathcal{A}$ is trimmable.

Suppose that a non-empty class $\mathcal{C}$ of connected graphs is trimmable. Then from what we saw about trees we have $\liminf \operatorname{in}_{n}\left(\tau\left(\mathcal{C}_{n}\right) / n !\right)^{1 / n} \geqslant \lambda e$. Let $\mathcal{B}=\mathcal{C}^{\delta \geqslant 2}$. Then $C(x, \tau)=B\left(\nu^{-1} T^{o}(x, \tau), \tau\right)$, where $C, B$ and $T^{o}$ are the exponential generating functions for $\mathcal{C}, \mathcal{B}$ and the rooted trees, respectively. Now if $0<x<(\lambda e)^{-1}$ then $\nu^{-1} T^{o}(x, \tau)<\lambda^{-1}$ by the last result in Section 2.2. Hence if $\rho(\mathcal{B}, \tau) \geqslant \lambda^{-1}$ then $\rho(\mathcal{C}, \tau) \geqslant(\lambda e)^{-1}$, and so $(\mathcal{C}, \tau)$ has growth constant $\lambda e$. We extend this observation below.

Lemma 5.2. Let the non-empty class $\mathcal{C}$ of connected graphs be trimmable, and let $\mathcal{B}=$ $\mathcal{C}^{\delta \geqslant 2}$. Suppose that either $(a)(\mathcal{B}, \tau)$ has growth constant $\beta$ and $\beta>\lambda$, in which case we 
let $\gamma=\beta e^{\lambda / \beta}$ (which is $>\lambda e$ ) and let $\alpha=1-\lambda / \beta$; or $(b)(\mathcal{B}, \tau)$ has radius of convergence $\geqslant \lambda^{-1}$ in which case we let $\gamma=\lambda$ e and let $\alpha=0$.

Then $(\mathcal{C}, \tau)$ is smooth, with growth constant $\gamma$. Further, let $R_{n}^{\mathcal{C}} \in_{\tau} \mathcal{C}$ : then for any $\epsilon>0$

$$
\mathbb{P}\left[\left|v\left(2-\operatorname{core}\left(R_{n}^{\mathcal{C}}\right)\right)-\alpha n\right|>\epsilon n\right]=e^{-\Omega(n)} .
$$

Proof. If $\mathcal{B}$ is empty then $\mathcal{C}$ is the class $\mathcal{T}$ of all trees, and we saw in Section 3.1 above that $(\mathcal{T}, \tau)$ is smooth with growth constant $\lambda e$ (and $\beta=0, \gamma=\lambda e$ and $\alpha=0$ ). Thus we may assume that $\mathcal{B}$ is non-empty. For $3 \leqslant k \leqslant n$ let

$$
f(n, k)=\nu \sum\left\{\lambda^{e(G)}: G \in \mathcal{C}_{n}, v(2-\operatorname{core}(G))=k\right\}
$$

Observe that $\left|\mathcal{C}_{1}\right|=1$ if the one-vertex graph $K_{1}$ is in $\mathcal{C}$, and $\left|\mathcal{C}_{1}\right|=0$ otherwise. The main idea of the proof was inspired by [2], and goes as follows for case (a), when $(\mathcal{B}, \tau)$ has growth constant $\gamma>\lambda e$. (We consider the case (b) later.) We first show that

$$
f(n, k)=(1+o(1))^{n} n ! \gamma^{n}
$$

when $k=(\alpha+o(1)) n$, and the expression on the right side gives an asymptotic approximation for $\tau\left(\mathcal{C}_{n}\right)$. Further, the dominant contribution in the sum

$$
\tau\left(\mathcal{C}_{n}\right)=\sum_{k=3}^{n} f(n, k)+\left|\mathcal{C}_{1}\right| n^{n-2} \lambda^{n-1} \nu
$$

is from $k$ as above; for all such $k$

$$
\frac{f(n+1, k)}{(n+1) f(n, k)} \sim \gamma
$$

and this yields

$$
\frac{\tau\left(\mathcal{C}_{n+1}\right)}{(n+1) \tau\left(\mathcal{C}_{n}\right)} \sim \gamma .
$$

Now for the details. Let us not yet assume that $\mathcal{B}$ has a growth constant (so that we can re-use the argument later). Recall that the number of forests on $[n]$ consisting of $k$ trees where vertices $1, \ldots, k$ are all in different trees is $k n^{n-1-k}$, see for example Theorem 3.3 of [54]. Thus

$$
f(n, k)=\left(\begin{array}{l}
n \\
k
\end{array}\right) \tau\left(\mathcal{B}_{k}\right) \lambda k(\lambda n)^{n-1-k} .
$$

Of course $f(n, n)=\tau\left(\mathcal{B}_{n}\right)$. We aim next to prove the three results (22), (23) and (24) below. We first consider case (a), and then case (b) will follow easily.

Suppose then that $\beta>0$. Let $r(n)=\frac{\tau\left(\mathcal{B}_{n}\right)}{n ! \beta^{n}}$ (so that we will have $r(n)=(1+o(1))^{n}$ below once we assume that $(\mathcal{B}, \tau)$ has growth constant $\beta$ ). Let $s(n)=\frac{n^{n}}{n ! e^{n}}$, so that by 
Stirling's formula $s(n) \sim(2 \pi n)^{-\frac{1}{2}}$, and $s(n) \leqslant 1$ for all $n$. Then for $3 \leqslant k \leqslant n-1$, writing $\kappa=k / n$,

$$
\begin{aligned}
f(n, k) & =n ! \frac{\tau\left(\mathcal{B}_{k}\right)}{k !} \frac{\lambda k}{\lambda n}\left(\frac{\lambda n}{n-k}\right)^{n-k} \frac{(n-k)^{n-k}}{(n-k) !} \\
& =n ! r(k) \beta^{k} \frac{k}{n}\left(\frac{\lambda e}{1-k / n}\right)^{n-k} s(n-k) \\
& =n ! \beta^{n}\left(\frac{\lambda e}{\beta(1-\kappa)}\right)^{(1-\kappa) n} \cdot \kappa r(k) s(n-k) \\
& =n ! \beta^{n}(h(1-\kappa))^{n} \cdot \kappa r(k) s(n-k)
\end{aligned}
$$

where $h(x)=\left(\frac{\lambda e}{\beta x}\right)^{x}$ for $x>0$ and $h(0)=1$. Thus (without yet assuming that $(\mathcal{B}, \tau)$ has a growth constant) we have

$$
f(n, k)=n ! \beta^{n}(h(1-\kappa))^{n} \cdot \kappa r(k) s(n-k) .
$$

Note that the function $h(x)$ strictly increases up to $x=\lambda / \beta$, where it has value $e^{\lambda / \beta}$, and strictly decreases above $\lambda / \beta$. Hence

$$
f(n, k) \leqslant n ! \beta^{n}\left(e^{\lambda / \beta}\right)^{n} r(k)
$$

and recalling that $\gamma=\beta e^{\lambda / \beta}$ we have

$$
f(n, k) \leqslant n ! \gamma^{n} r(k) .
$$

(We will use this inequality in the proof of lemma 5.3.)

Now assume that $(\mathcal{B}, \tau)$ has growth constant $\beta$, so that $g(n)=(1+o(1))^{n}$ by the definition of the growth constant. Then from (19), uniformly over $k$ with $3 \leqslant k \leqslant n$, still writing $\kappa=k / n$, we have

$$
f(n, k)=(1+o(1))^{n} n ! \beta^{n}(h(1-\kappa))^{n} .
$$

We need to consider two subcases.

(i) Suppose first that $\beta>\lambda$, so $\gamma=\beta e^{\lambda / \beta}$ and $\alpha=1-\lambda / \beta$. Then it follows from (17), (21) and the properties of $h$ that

$$
\tau\left(\mathcal{C}_{n}\right)=(1+o(1))^{n} n ! \gamma^{n}
$$

(the possible term $n^{n-2} \lambda^{n-1} \nu$ in the sum (17) is negligible since $\gamma>\lambda e$ ), and for any $\delta>0$ there exists $\eta>0$ such that

$$
\sum_{k:|k-\alpha n| \geqslant \delta n} f(n, k) \leqslant(1-\eta+o(1))^{n} n ! \gamma^{n} .
$$

Thus for any $\delta>0$

$$
\frac{\tau\left(\mathcal{C}_{n+1}\right)}{(n+1) \tau\left(\mathcal{C}_{n}\right)} \sim \frac{\sum_{k:|k-\alpha n|<\delta n} f(n+1, k)}{\sum_{k:|k-\alpha n|<\delta n}(n+1) f(n, k)}
$$


(ii) If $\beta=\lambda$ then $\gamma=\lambda e$ and $\alpha=0$, and the results (22), (23) and (24) follow as above.

Finally consider the case $\rho(\mathcal{B}, \tau) \geqslant \lambda^{-1}$. We shall see that we have exactly the same results $(22),(23)$ and $(24)$ as for the case (ii). We know that $\tau\left(\mathcal{C}_{n}\right) \geqslant(1+o(1))^{n} n !(\lambda e)^{n}$. Also, we may add connected graphs to $\mathcal{C}$, maintaining trimmability, to form $\mathcal{C}^{\prime}$ so that if $\mathcal{B}^{\prime}$ denotes $\left\{G \in \mathcal{C}^{\prime}: \delta(G) \geqslant 2\right\}$ then $\left(\mathcal{B}^{\prime}, \tau\right)$ has growth constant $\lambda$. Thus from (22) and (23) for $\mathcal{C}^{\prime}$ and $\mathcal{B}^{\prime}$ we obtain the corresponding results for $\mathcal{C}$ and $\mathcal{B}$ in this case, and then we may deduce (24). We have now established (22), (23) and (24) for both cases (a) and (b).

By equation (18), for each $k$ such that $3 \leqslant k \leqslant n$ and $\mathcal{B}_{k} \neq \emptyset$, writing $k=\kappa n$ we have

$$
\frac{f(n+1, k)}{(n+1) f(n, k)}=\frac{\lambda n}{n+1-k}\left(1+\frac{1}{n}\right)^{n-k}=\frac{\lambda\left(1+\frac{1}{n}\right)^{(1-\kappa) n}}{1+\frac{1}{n}-\kappa} .
$$

Now $\lambda \frac{e^{1-\alpha}}{1-\alpha}=\beta e^{\lambda / \beta}=\gamma$ if $\beta>\lambda$, and $\lambda \frac{e^{1-\alpha}}{1-\alpha}=\lambda e=\gamma$ if $\beta=\lambda$. Let $\epsilon>0$. By considering the two cases for $\beta$, we see that there exist $n_{0}$ and $\delta>0$ such that whenever $n \geqslant n_{0}$ and $|\kappa-\alpha|<\delta$ we have

$$
(1-\epsilon) \gamma \leqslant \frac{\lambda\left(1+\frac{1}{n}\right)^{(1-\kappa) n}}{1+\frac{1}{n}-\kappa} \leqslant(1+\epsilon) \gamma
$$

Hence by (24) we have

$$
(1-\epsilon+o(1)) \gamma \leqslant \frac{\tau\left(\mathcal{C}_{n+1}\right)}{(n+1) \tau\left(\mathcal{C}_{n}\right)} \leqslant(1+\epsilon+o(1)) \gamma .
$$

Thus

$$
\frac{\tau\left(\mathcal{C}_{n+1}\right)}{(n+1) \tau\left(\mathcal{C}_{n}\right)} \rightarrow \gamma \quad \text { as } n \rightarrow \infty
$$

as required. The last part of the lemma, concerning the size of the core, follows directly from (22) and (23).

Lemma 5.3. Let the class $\mathcal{C}$ of connected graphs be trimmable, and suppose that $(\mathcal{C}, \tau)$ has growth constant $\gamma$; and let $\mathcal{B}=\mathcal{C}^{\delta \geqslant 2}$.

If $\gamma>\lambda$ e and $(\mathcal{B}, \tau)$ maintains at least factorial growth then $(\mathcal{B}, \tau)$ has growth constant $\beta$ where $\beta$ is the unique root $>\lambda$ of $\beta e^{\lambda / \beta}=\gamma$. If $\gamma \leqslant \lambda$ e then $\rho(\mathcal{B}, \tau) \geqslant \beta^{-1}$ where $\beta=\lambda$.

Proof. We prove first that $\lim \sup \left(\tau\left(\mathcal{B}_{n}\right) / n !\right)^{1 / n} \leqslant \beta$, by showing that otherwise equation (19) in the proof of the last result will yield $\lim \sup \left(\tau\left(\mathcal{C}_{n}\right) / n !\right)^{1 / n}>\gamma$. Let $\hat{\beta}>\beta$. If $\tau\left(\mathcal{B}_{k}\right) \geqslant k ! \hat{\beta}^{k}$ and we let $n=\left\lceil\frac{k}{1-(\lambda / \hat{\beta})}\right\rceil$ then $h(1-k / n) \sim h(\lambda / \hat{\beta})=e^{\lambda / \hat{\beta}}$, and so by $(19)$ we have

$$
\left(\frac{\tau\left(\mathcal{C}_{n}\right)}{n !}\right)^{\frac{1}{n}} \geqslant\left(\frac{f(n, k)}{n !}\right)^{\frac{1}{n}} \geqslant(1+o(1)) \hat{\beta} e^{\lambda / \hat{\beta}}
$$


But the function $f(x)=x \ln \lambda / x$ is strictly increasing for $x>\lambda$, so $\hat{\beta} e^{\lambda / \hat{\beta}}>\beta e^{\lambda / \beta}=\gamma$. Thus $\lim \sup \left(\tau\left(\mathcal{C}_{n}\right) / n !\right)^{1 / n}>\gamma$, which contradicts the assumption that $(\mathcal{C}, \tau)$ has growth constant $\gamma$.

For the case when $\gamma>\lambda e$ we also need a lower bound. Let $0<\epsilon<1$. We want to show that for all sufficiently large $n$ we have

$$
\tau\left(\mathcal{B}_{n}\right) \geqslant n ! \beta^{n}(1-\epsilon)^{n}
$$

We now use the assumption that $(\mathcal{B}, \tau)$ maintains at least factorial growth (in the form in Lemma 3.6). Let $\delta^{\prime}>0, \eta>0$ and $g(n)=(1+o(1))^{n}$ be such that for each $n$ and each $j$ with $1 \leqslant j \leqslant \delta^{\prime} n$ we have

$$
\tau\left(\mathcal{B}_{n}\right) \geqslant \tau\left(\mathcal{B}_{n-j}\right)(n)_{j} \eta^{j} g(n)
$$

Let $0<\delta<\min \left\{\delta^{\prime}, 1-(\lambda / \beta)\right\}$ be sufficiently small that $(\eta / \beta)^{\delta} \geqslant 1-\epsilon / 3$. We claim that there is an $n_{0}$ such that for all $n \geqslant n_{0}$ there is an $\tilde{n}$ with $|n-\tilde{n}|<\delta n$ such that

$$
\tau\left(\mathcal{B}_{\tilde{n}}\right) \geqslant \tilde{n} ! \beta^{\tilde{n}}(1-\epsilon / 3)^{\tilde{n}}
$$

The idea is that the proof of the last result, Lemma 5.2, shows that there must be such an $n_{0}$ since otherwise $\tau\left(\mathcal{C}_{n}\right)$ would be too small for each large $n$. For suppose there is no such $n_{0}$. Then for arbitrarily large values of $k$, each $j$ with $|j-k| \leqslant \delta k$ has $\tau\left(\mathcal{B}_{j}\right)<j ! \beta^{j}(1-\epsilon / 3)^{j}$; that is, $r(j)<(1-\epsilon / 3)^{j}$, where $r(j)=\frac{\tau\left(\mathcal{B}_{j}\right)}{j ! \beta^{j}}$ as in the proof of the last lemma. Consider such a $k$, and let $n=\left\lceil\frac{k}{1-(\lambda / \beta)}\right\rceil$ as above. As we saw in (23) above, there is a constant $\eta>0$ such that

$$
\sum_{j:|j-k| \geqslant \delta k} f(n, j) \leqslant n ! \gamma^{n}(1-\eta+o(1))^{n},
$$

and now also by (20)

$$
\sum_{j:|j-k|<\delta k} f(n, j) \leqslant n ! \gamma^{n} \sum_{j:|j-k|<\delta k} r(j) \leqslant c \cdot n ! \gamma^{n}(1-\epsilon / 3)^{(1-(\lambda / \beta)-\delta) n} .
$$

for a suitable constant $c$. Let $\eta^{\prime}>0$ satisfy $\eta^{\prime}<\eta$ and $1-\eta^{\prime}>(1-\epsilon / 3)^{1-(\lambda / \beta)-\delta}$. Then by (17) and the above

$$
\left(\frac{\tau\left(\mathcal{C}_{n}\right)}{n !}\right)^{\frac{1}{n}} \leqslant\left(1-\eta^{\prime}\right) \gamma
$$

if $n$ is sufficiently large. This contradicts the assumption that $(\mathcal{C}, \tau)$ has growth constant $\gamma$, and completes the proof of (26). Indeed we can insist that there is a value $\tilde{n}$ as above with $\tilde{n} \leqslant n \leqslant(1+\delta) \tilde{n}$. To see this we may apply to $n-\lfloor\delta n / 2\rfloor$ the current version of $(26)$ with $\delta$ replaced by $\delta / 2$.

Let $n_{1} \geqslant 2 n_{0}$ be such that $g(n) \geqslant(1-\epsilon / 3)^{n}$ for all $n \geqslant n_{1}$. Let $n \geqslant n_{1}$. It will suffice for us to show that (25) holds for $n$. Let $j=n-\tilde{n}$. If $j=0$ there is nothing to prove so 
we may assume that $1 \leqslant j \leqslant \delta n$. Then

$$
\begin{aligned}
\tau\left(\mathcal{B}_{n}\right) & \geqslant \tau\left(\mathcal{B}_{\tilde{n}}\right)(n)_{j} \eta^{j} g(n) \\
& \geqslant n ! \beta^{n} \beta^{-j}(1-\epsilon / 3)^{n} \eta^{j} g(n) \\
& \geqslant n ! \beta^{n}(\eta / \beta)^{j}(1-\epsilon / 3)^{2 n} \\
& \geqslant n ! \beta^{n}(1-\epsilon / 3)^{3 n} \geqslant n ! \beta^{n}(1-\epsilon)^{n}
\end{aligned}
$$

as required.

\subsection{Proof of Theorem 2.4}

The weighted class $(\mathcal{C}, \tau)$ has the same growth constant $\gamma$ as $(\mathcal{A}, \tau)$, for example since $\mathcal{A}$ is bridge-addable. Let $\mathcal{B}=\mathcal{C}^{\delta \geqslant 2}$. If $\gamma>\lambda e$ then, since $\mathcal{C}$ is trimmable, by Lemma 5.3 $(\mathcal{B}, \tau)$ has growth constant $\beta$. If $\gamma \leqslant \lambda e$ then $\rho(\mathcal{B}, \tau) \geqslant \lambda^{-1}$. But now (without restriction on $\gamma$ ) Lemma 5.2 shows that $(\mathcal{C}, \tau)$ is smooth, and further shows that, for $R_{n}^{\mathcal{C}} \in_{\tau} \mathcal{C}$, we have for any $\epsilon>0$ that

$$
\mathbb{P}\left(\left|v\left(2-\operatorname{core}\left(R_{n}^{\mathcal{C}}\right)\right)-\alpha n\right|>\epsilon n\right)=e^{-\Omega(n)} .
$$

Also, for $R_{n}^{\mathcal{A}} \in_{\tau} \mathcal{A}, \mathbb{P}\left(\operatorname{Frag}\left(R_{n}^{\mathcal{A}}\right) \in \mathcal{F}_{\mathcal{A}}\right)=1-o(1)$ by Lemma 3.4. We may now use Lemma 5.1 to show that $(\mathcal{A}, \tau)$ is smooth. At this point we have proved $(27)$ and parts (a) and (b) of Theorem 2.4.

Next we prove part (c). Observe that conditional on $\operatorname{big}\left(R_{n}^{\mathcal{A}}\right)=n^{\prime}$ the distribution of $\operatorname{Big}\left(R_{n}^{\mathcal{A}}\right)$ is the same as that of $R_{n^{\prime}}^{\mathcal{C}}$. Thus for each $j<n$ and each $t$

$$
\begin{aligned}
& \mathbb{P}\left(v\left(2-\operatorname{core}\left(R_{n-j}^{\mathcal{C}}\right)\right) \geqslant t\right) \\
\leqslant & \mathbb{P}\left(v\left(2-\operatorname{core}\left(R_{n}^{\mathcal{A}}\right)\right) \geqslant t \mid \operatorname{frag}\left(R_{n}^{\mathcal{A}}\right)=j\right) \leqslant \mathbb{P}\left(v\left(2-\operatorname{core}\left(R_{n-j}^{\mathcal{C}}\right)\right) \geqslant t-j\right) .
\end{aligned}
$$

Let $\epsilon>0$. Let $\omega=\omega(n)=\lfloor\epsilon n / 2\rfloor$. Then $\mathbb{P}\left(v\left(2-\operatorname{core}\left(R_{n}^{\mathcal{A}}\right)\right) \geqslant(\alpha+\epsilon) n\right)$ is at most

$$
\mathbb{P}\left(\left(v\left(2-\operatorname{core}\left(R_{n}^{\mathcal{A}}\right)\right) \geqslant(\alpha+\epsilon) n\right) \cap\left(\operatorname{frag}\left(R_{n}^{\mathcal{A}}\right) \leqslant \omega\right)\right)+\mathbb{P}\left(\operatorname{frag}\left(R_{n}^{\mathcal{A}}\right)>\omega\right) .
$$

By Lemma 3.1 (b) we know that the second term $\mathbb{P}\left(\operatorname{frag}\left(R_{n}^{\mathcal{A}}\right)>\omega\right)$ is $o(1)$. But the first term equals

$$
\begin{aligned}
& \sum_{j=0}^{\omega} \mathbb{P}\left(\left(v\left(2-\operatorname{core}\left(R_{n}^{\mathcal{A}}\right)\right) \geqslant(\alpha+\epsilon) n\right) \cap\left(\operatorname{frag}\left(R_{n}^{\mathcal{A}}\right)=j\right)\right) \\
\leqslant & \sum_{j=0}^{\omega} \mathbb{P}\left(v\left(2-\operatorname{core}\left(R_{n-j}^{\mathcal{C}}\right)\right) \geqslant(\alpha+\epsilon) n-j\right)=e^{-\Omega(n)}
\end{aligned}
$$

Thus

$$
\mathbb{P}\left(v\left(2-\operatorname{core}\left(R_{n}^{\mathcal{A}}\right)\right) \geqslant(\alpha+\epsilon) n\right)=o(1)
$$


Similarly

$$
\begin{aligned}
& \mathbb{P}\left(v\left(2-\operatorname{core}\left(R_{n}^{\mathcal{A}}\right)\right) \leqslant(\alpha-\epsilon) n\right) \\
& \quad \leqslant \sum_{j=0}^{\omega} \mathbb{P}\left(v\left(2-\operatorname{core}\left(R_{n-j}^{\mathcal{C}}\right)\right) \leqslant(\alpha-\epsilon) n\right)+\mathbb{P}\left(\operatorname{frag}\left(R_{n}^{\mathcal{A}}\right)>\omega\right)=o(1) .
\end{aligned}
$$

Now consider the remaining part of the theorem, part (d), and assume that $\gamma>\lambda e$. Note first that is it very unlikely that $2-\operatorname{core}\left(R_{n}\right)$ is empty; for this happens (if and) only if $R_{n}$ is a forest, and the probability of this happening is $(\lambda e / \gamma+o(1))^{n}=e^{-\Omega(n)}$. Also, the probability that $\operatorname{Big}\left(R_{n}\right)$ is a tree is $e^{-\Omega(n)}$. For

$$
\begin{aligned}
& \mathbb{P}\left(\operatorname{Big}\left(R_{n}\right) \text { is a tree and } \operatorname{big}\left(R_{n}\right) \geqslant \frac{2}{3} n\right) \\
& \qquad \sum_{\frac{2}{3} n \leqslant a \leqslant n}\left(\begin{array}{l}
n \\
a
\end{array}\right) \frac{\tau\left(\mathcal{T}_{a}\right) \cdot \tau\left(\mathcal{A}_{n-a}\right)}{\tau\left(\mathcal{A}_{n}\right)}=\sum_{\frac{2}{3} n \leqslant a \leqslant n} \frac{\frac{\tau\left(\mathcal{T}_{a}\right)}{a !} \cdot \frac{\tau\left(\mathcal{A}_{n-a}\right)}{(n-a) !}}{\frac{\tau\left(\mathcal{A}_{n}\right)}{n !}} \\
& =(1+o(1))^{n} \sum_{\frac{2}{3} n \leqslant a \leqslant n} \frac{(\lambda e)^{a} \gamma^{n-a}}{\gamma^{n}}=(1+o(1))^{n}(\lambda e / \gamma)^{\frac{2}{3} n}=e^{-\Omega(n)} .
\end{aligned}
$$

But if $2-\operatorname{core}\left(R_{n}\right)$ is non-empty and $\operatorname{Big}\left(R_{n}\right)$ is not a tree, then $2-\operatorname{core}\left(R_{n}\right)$ is connected if and only if $\operatorname{Frag}\left(R_{n}\right)$ is acyclic. Thus

$$
\mid \mathbb{P}\left(2-\operatorname{core}\left(R_{n}\right) \text { connected }(\text { and } \neq \emptyset)\right)-\mathbb{P}\left(\text { Frag }\left(R_{n}\right) \text { acyclic }\right) \mid=e^{-\Omega(n)} .
$$

Finally, the probability that $\operatorname{Frag}\left(R_{n}^{\mathcal{A}}\right)$ has no non-tree components tends to $e^{-(D(\rho, \tau)-T(\rho, \tau))}$ by Corollary 2.3 (b) applied to $\mathcal{D} \backslash \mathcal{T}$.

\section{Poisson convergence}

Let $\tau=(\lambda, \nu)$ and $\rho>0$ be given. As in (5), we use the notation

$$
\mu(H)=\rho^{v(H)} \lambda^{e(H)} \nu^{\kappa(H)} / \operatorname{aut}(H) \text { for each graph } H .
$$

Also, let $r_{n}=n \tau\left(\mathcal{A}_{n-1}\right) / \tau\left(\mathcal{A}_{n}\right)$, and assume that $\mathcal{A}_{n} \neq \emptyset$ when necessary. Further, recall the notation $(n)_{k}=n(n-1) \cdots(n-k+1)$.

The following lemma is a slight extension for example of Lemma 4.1 of [48]. It will be a key result for taking advantage of smoothness. Given a graph $G$ and a connected graph $H$ we let $\kappa(G, H)$ be the number of components of $G$ isomorphic to $H$.

Lemma 6.1. Let $\mathcal{A}$ be any class of graphs, and let $\tau$ and $\rho>0$ be given. Let $H_{1}, \ldots, H_{h}$ be pairwise non-isomorphic connected graphs, each freely addable to $\mathcal{A}$. Let $k_{1}, \ldots, k_{h}$ be non-negative integers, and let $K=\sum_{i=1}^{h} k_{i} v\left(H_{i}\right)$. Then for $R_{n} \in_{\tau} \mathcal{A}$

$$
\mathbb{E}\left[\prod_{i=1}^{h}\left(\kappa\left(R_{n}, H_{i}\right)\right)_{k_{i}}\right]=\prod_{i=1}^{h} \mu\left(H_{i}\right)^{k_{i}} \cdot \prod_{j=1}^{K}\left(r_{n-j+1} / \rho\right) .
$$


Proof. We may construct a graph $G$ in $\mathcal{A}_{n}$ with $\kappa\left(G, H_{i}\right) \geqslant k_{i}$ for each $i$ as follows: choose a list of $K$ vertices; put a copy of $H_{1}$ on the first $v\left(H_{1}\right)$ vertices in the list, if $k_{1}>1$ put another copy of $H_{1}$ on the next $v\left(H_{1}\right)$ vertices, and so on until we put a copy of $H_{h}$ on the last $v\left(H_{h}\right)$ vertices in the list; and finally put any graph of order $n-K$ in $\mathcal{A}$ on the remaining $n-K$ vertices. The sum over all such constructions of the weight $\lambda^{e(G)} \nu^{\kappa(G)}$ of the graph $G$ constructed is

$$
(n)_{K} \prod_{i=1}^{h}\left(\operatorname{aut}\left(H_{i}\right)^{-1} \lambda^{e\left(H_{i}\right)} \nu\right)^{k_{i}} \cdot \tau\left(\mathcal{A}_{n-K}\right) .
$$

Now observe that each graph $G \in \mathcal{A}_{n}$ is constructed $\prod_{i=1}^{h}\left(\kappa\left(G, H_{i}\right)\right)_{k_{i}}$ times (exactly); and so the above expression equals

$$
\sum_{G \in \mathcal{A}_{n}} \prod_{i=1}^{h}\left(\kappa\left(G, H_{i}\right)\right)_{k_{i}} \lambda^{e(G)} \nu^{\kappa(G)} .
$$

But by definition $\mathbb{E}\left[\prod_{i=1}^{m}\left(\kappa\left(R_{n}, H_{i}\right)\right)_{k_{i}}\right]$ is $\tau\left(\mathcal{A}_{n}\right)^{-1}$ times this last quantity. Hence

$$
\begin{aligned}
\mathbb{E}\left[\prod_{i=1}^{m}\left(\kappa\left(R_{n}, H_{i}\right)\right)_{k_{i}}\right] & =(n)_{K} \prod_{i=1}^{h}\left(\operatorname{aut}\left(H_{i}\right)^{-1} \lambda^{e\left(H_{i}\right)} \nu\right)^{k_{i}} \cdot \tau\left(\mathcal{A}_{n-K}\right) / \tau\left(\mathcal{A}_{n}\right) \\
& =\prod_{i=1}^{h} \mu\left(H_{i}\right)^{k_{i}} \cdot \prod_{j=1}^{K}\left(\rho^{-1}(n-j+1) \frac{\tau\left(\mathcal{A}_{n-j}\right)}{\tau\left(\mathcal{A}_{n-j+1}\right)}\right) \\
& =\prod_{i=1}^{h} \mu\left(H_{i}\right)^{k_{i}} \cdot \prod_{j=1}^{K}\left(r_{n-j+1} / \rho\right)
\end{aligned}
$$

as required.

When we add the assumption that $(\mathcal{A}, \tau)$ is smooth, we find convergence of distributions. Recall that $\rho(\mathcal{A}, \tau)$ denotes the radius of convergence of $A(x, \tau)$ as a power series in $x$.

Lemma 6.2. Let the weighted graph class $(\mathcal{A}, \tau)$ be smooth, and let $\rho=\rho(\mathcal{A}, \tau)$. Let $H_{1}, \ldots, H_{h}$ be a fixed family of pairwise non-isomorphic connected graphs, each freely addable to $\mathcal{A}$. Then as $n \rightarrow \infty$ the joint distribution of the random variables $\kappa\left(R_{n}, H_{1}\right)$, $\ldots, \kappa\left(R_{n}, H_{h}\right)$ converges in total variation to the product distribution $\operatorname{Po}\left(\mu\left(H_{1}\right)\right) \otimes \cdots \otimes$ $\operatorname{Po}\left(\mu\left(H_{h}\right)\right)$.

Proof. Since $r_{n} \rightarrow \rho$ as $n \rightarrow \infty$, by the last lemma

$$
\mathbb{E}\left[\prod_{i=1}^{h}\left(\kappa\left(R_{n}, H_{i}\right)\right)_{k_{i}}\right] \rightarrow \prod_{i=1}^{h} \mu\left(H_{i}\right)^{k_{i}}
$$


as $n \rightarrow \infty$, for all non-negative integers $k_{1}, \ldots, k_{h}$. A standard result on the Poisson distribution now shows the joint distribution of the random variables $\kappa\left(R_{n}, H_{1}\right), \ldots, \kappa\left(R_{n}, H_{h}\right)$ tends to that of independent random variables $\operatorname{Po}\left(\tau\left(H_{1}\right)\right), \ldots, \operatorname{Po}\left(\tau\left(H_{h}\right)\right)$, see for example Theorem 6.10 of Janson, Łuczak and Ruciński [39]. Thus for each $h$-tuple of non-negative integers $\left(t_{1}, \ldots, t_{h}\right)$

$$
\mathbb{P}\left[\kappa\left(R_{n}, H_{i}\right)=t_{i} \forall i\right] \rightarrow \prod_{i} \mathbb{P}\left[\kappa\left(R_{n}, H_{i}\right)=t_{i}\right] \quad \text { as } n \rightarrow \infty
$$

and so we have pointwise convergence of probabilities, which is equivalent to convergence in total variation.

\section{$7 \operatorname{Frag}\left(R_{n}\right)$ and connectivity}

The following lemma parallels Lemma 5.1, which showed that, under suitable conditions, if the weighted class $(\mathcal{C}, \tau)$ of connected graphs in $\mathcal{A}$ is smooth then $(\mathcal{A}, \tau)$ is smooth. The lemma below shows the converse result that if $(\mathcal{A}, \tau)$ is smooth then, for $R_{n} \in_{\tau} \mathcal{A}$ the probability that $R_{n}$ is connected tends to a limit, and so $(\mathcal{C}, \tau)$ is smooth.

Lemma 7.1. Let the graph class $\mathcal{A}$ be bridge-addable; let $\rho=\rho(\mathcal{A}, \tau)$; let $\mathcal{F}_{\mathcal{A}}$ denote the class of graphs freely addable to $\mathcal{A}$; and suppose that, for $R_{n} \in_{\tau} \mathcal{A}, w h p \operatorname{Frag}\left(R_{n}\right) \in \mathcal{F}_{\mathcal{A}}$. Let $\mathcal{C}$ and $\mathcal{D}$ be the classes of connected graphs in $\mathcal{A}$ and $\mathcal{F}_{\mathcal{A}}$ respectively, and let $F_{\mathcal{A}}$ denote the exponential generating function of $\mathcal{F}_{\mathcal{A}}$. Suppose that $(\mathcal{A}, \tau)$ is smooth. Then $F_{\mathcal{A}}(\rho, \tau)$ and $D(\rho, \tau)$ are finite; $\kappa\left(R_{n}\right) \rightarrow_{T V} 1+\operatorname{Po}(D(\rho, \tau))$; and in particular

$$
\operatorname{Pr}\left[R_{n} \text { is connected }\right] \rightarrow e^{-D(\rho, \tau)}=1 / F_{\mathcal{A}}(\rho, \tau) \text { as } n \rightarrow \infty,
$$

and so $(\mathcal{C}, \tau)$ is smooth.

Proof. We follow the method of proof of Lemma 4.3 of [48]. We first show that $D(\rho, \tau)$ is finite. By Lemma 3.1 (b) we may choose a (fixed) $k$ sufficiently large that $\mathbb{P}\left[\operatorname{frag}\left(R_{n}\right) \geqslant\right.$ $k] \leqslant \frac{1}{3}$ for all $n$, and $\mathbb{P}[\operatorname{Po}(2 k) \geqslant k] \geqslant \frac{2}{3}$. Suppose that $D(\rho, \tau) \geqslant 2 k+1$. Then by $(4)$ there are distinct $H_{1}, \ldots, H_{m}$ in $\mathcal{U D}$ such that $\sum_{i=1}^{m} \mu\left(H_{i}\right)=\mu_{0} \geqslant 2 k$. It follows by Lemma 6.2 that, for $n>k \max _{i} v\left(H_{i}\right)$

$$
\frac{1}{3} \geqslant \mathbb{P}\left[\operatorname{frag}\left(R_{n}\right) \geqslant k\right] \geqslant \mathbb{P}\left[\sum_{i=1}^{m} \kappa\left(R_{n}, H_{i}\right) \geqslant k\right] \rightarrow \mathbb{P}\left[\operatorname{Po}\left(\mu_{0}\right) \geqslant k\right] \geqslant \frac{2}{3}
$$

as $n \rightarrow \infty$, a contradiction. Hence $D(\rho, \tau)$ is finite, and so $F_{\mathcal{A}}(\rho, \tau)$ is finite too.

Now let $\mu=D(\rho, \tau)$. Let $k$ be a fixed positive integer and let $\epsilon>0$. We want to show that for $n$ sufficiently large we have

$$
\left|\mathbb{P}\left[\kappa\left(\operatorname{Frag}\left(R_{n}\right)\right)=k\right]-\mathbb{P}[\operatorname{Po}(\mu)=k]\right|<\epsilon .
$$

By our assumptions, there is an $n_{0}$ such that for each $n \geqslant n_{0}$

$$
\mathbb{P}\left[\operatorname{frag}\left(R_{n}\right)>n_{0}\right]+\mathbb{P}\left[\operatorname{Frag}\left(R_{n}\right) \notin \mathcal{F}_{\mathcal{A}}\right]<\epsilon / 3 .
$$


List the graphs in $\mathcal{U D}$ in non-decreasing order of the number of vertices as $H_{1}, H_{2}, \ldots$ For each positive integer $m$ let $\mu^{(m)}=\sum_{i=1}^{m} \mu\left(H_{i}\right)$. Note that $D(\rho, \tau)=\sum_{H \in \mathcal{U D}} \mu(H)$ by (4) applied to $\mathcal{D}$. Thus we may choose $n_{1} \geqslant n_{0}$ such that, if $m$ is the largest index such that $v\left(H_{m}\right) \leqslant n_{1}$, then

$$
\left|\mathbb{P}[\operatorname{Po}(\mu)=k]-\mathbb{P}\left[\operatorname{Po}\left(\mu^{(m)}\right)=k\right]\right|<\epsilon / 3 .
$$

Observe that for any graph $G$ with more than $2 n_{1}$ vertices, if $\operatorname{frag}(G) \leqslant n_{0}$ and $\operatorname{Frag}(G) \in$ $\mathcal{F}_{\mathcal{A}}$, then $\kappa(\operatorname{Frag}(G))$ is the number of components of $G$ isomorphic to one of $H_{1}, \ldots, H_{m}$ (that is, with order at most $n_{1}$ ). Let $X_{n}$ denote the number of components of $R_{n}$ isomorphic to one of $H_{1}, \ldots, H_{m}$. Let $n>2 n_{1}$. Then

$$
\left|\mathbb{P}\left[\kappa\left(\operatorname{Frag}\left(R_{n}\right)\right)=k\right]-\mathbb{P}\left[X_{n}=k\right]\right| \leqslant \mathbb{P}\left[\operatorname{frag}\left(R_{n}\right)>n_{0}\right]+\mathbb{P}\left[\operatorname{Frag}\left(R_{n}\right) \notin \mathcal{F}_{\mathcal{A}}\right]<\epsilon / 3 .
$$

But by Lemma 6.2, for $n$ sufficiently large,

$$
\left|\mathbb{P}\left[X_{n}=k\right]-\mathbb{P}\left[\operatorname{Po}\left(\mu^{(m)}\right)=k\right]\right|<\epsilon / 3,
$$

and then by (30) and (31) the inequality (28) follows. Therefore we have shown that $\kappa\left(R_{n}\right) \rightarrow_{T V} 1+\operatorname{Po}(D(\rho, \tau))$, and in particular

$$
\tau\left(\mathcal{C}_{n}\right) / \tau\left(\mathcal{A}_{n}\right)=\mathbb{P}\left(R_{n} \text { is connected }\right) \rightarrow e^{-D(\rho, \tau)} \text { as } n \rightarrow \infty \text {. }
$$

Finally observe that since $(\mathcal{A}, \tau)$ is smooth, and $\tau\left(\mathcal{C}_{n}\right) / \tau\left(\mathcal{A}_{n}\right)$ tends to a non-zero limit as $n \rightarrow \infty$ (namely $e^{-D(\rho, \tau)}$ ), it follows that $(\mathcal{C}, \tau)$ is smooth.

The next lemma has similar premises to Lemma 7.1, and obtains further conclusions. We use the same notation.

Lemma 7.2. Let $\mathcal{A}$ be bridge-addable; let $\rho=\rho(\mathcal{A}, \tau)$, and suppose that $\operatorname{Frag}\left(R_{n}\right) \in \mathcal{F}_{\mathcal{A}}$ whp where $R_{n} \in_{\tau} \mathcal{A}$. Let $\mathcal{C}$ be the class of connected graphs in $\mathcal{A}$. Assume that either $(\mathcal{A}, \tau)$ or $(\mathcal{C}, \tau)$ is smooth. Then both $(\mathcal{A}, \tau)$ and $(\mathcal{C}, \tau)$ are smooth; $F_{\mathcal{A}}(\rho, \tau)$ is finite; and the unlabelled graph $F_{n}$ corresponding to $\operatorname{Frag}\left(R_{n}\right)$ satisfies $F_{n} \rightarrow_{T V} F$, where

$$
\mathbb{P}[F=H]=\frac{\mu(H)}{F_{\mathcal{A}}(\rho, \tau)} \quad \text { for each } H \in \mathcal{U F}_{\mathcal{A}}
$$

Proof. Lemmas 5.1 and 7.1 show that both $(\mathcal{A}, \tau)$ and $(\mathcal{C}, \tau)$ are smooth, and that $F_{\mathcal{A}}(\rho, \tau)$ is finite. Let $a_{n}=\tau\left(\mathcal{A}_{n}\right)$ and $c_{n}=\tau\left(\mathcal{C}_{n}\right)$. Let $\mathcal{D}$ be the class of connected graphs in $\mathcal{F}_{\mathcal{A}}$. By Lemma 7.1

$$
c_{n} / a_{n} \rightarrow e^{-D(\rho, \tau)}=1 / F_{\mathcal{A}}(\rho, \tau) \text { as } n \rightarrow \infty .
$$

Given a graph $G$ on a finite subset $V$ of the positive integers let $\phi(G)$ be the natural copy of $G$ moved down on to $\{1, \ldots,|V|\}$; that is, let $\phi(G)$ be the graph on $\{1, \ldots,|V|\}$ such that the increasing bijection between $V$ and $\{1, \ldots,|V|\}$ is an isomorphism between $G$ and $\phi(G)$. 
Let $H$ be any graph in $\mathcal{B}$ on $[h]$. Then

$$
\begin{aligned}
\mathbb{P}\left[\phi\left(\operatorname{Frag}\left(R_{n}\right)\right)=H\right] & =\left(\begin{array}{c}
n \\
h
\end{array}\right) \frac{c_{n-h}}{a_{n}}=\frac{c_{n-h}}{a_{n-h}} \frac{1}{h !} \frac{(n)_{h} a_{n-h}}{a_{n}} \\
& =\frac{c_{n-h}}{a_{n-h}} \frac{1}{h !} \prod_{i=0}^{h-1} r_{n-i} \rightarrow e^{-D(\rho, \tau)} \frac{\rho^{h}}{h !}
\end{aligned}
$$

as $n \rightarrow \infty$ by $(33)$ and the fact that $(\mathcal{A}, \tau)$ is smooth. Now by symmetry

$$
\mathbb{P}\left[F_{n} \cong H\right]=\frac{h !}{\operatorname{aut}(H)} \mathbb{P}\left[\phi\left(\operatorname{Frag}\left(R_{n}\right)\right)=H\right]
$$

and hence as $n \rightarrow \infty$

$$
\mathbb{P}\left[F_{n} \cong H\right] \rightarrow e^{-D(\rho, \tau)} \frac{\rho^{h}}{\operatorname{aut}(H)}=\operatorname{Pr}(F \cong H) .
$$

Thus for each $H \in \mathcal{U B}$, as $n \rightarrow \infty$ we have $\mathbb{P}\left[F_{n}=H\right] \rightarrow \mathbb{P}(F=H)$; that is, $F_{n} \rightarrow_{T V}$ $U$.

We need one last lemma to complete the proof of Theorem 2.2.

Lemma 7.3. Let the weighted graph class $(\mathcal{A}, \tau)$ be well-behaved; let $\mathcal{D}$ be the class of connected graphs in $\mathcal{F}_{\mathcal{A}}$, with generating function $D$; and let $\rho=\rho(\mathcal{A}, \tau)$. Then $D^{\prime}(\rho, \tau)$ is finite (where we are differentiating with respect to the first variable).

Proof. Note that $0<\rho<\infty$. By Lemma $3.1(\mathrm{~b}), \mathbb{E}\left[\operatorname{frag}\left(R_{n}\right)\right] \leqslant c$ for all $n$ where $c=2 \nu / \lambda$. Suppose that $D^{\prime}(\rho, \tau) \geqslant(c+3) / \rho$. Then by (4) applied to $\mathcal{D}$, there are distinct $H_{1}, \ldots, H_{m}$ in $\mathcal{U D}$ such that $\sum_{i=1}^{m} v\left(H_{i}\right) \mu\left(H_{i}\right)=\alpha \geqslant c+2$. Let $n_{0}=\max _{i} v\left(H_{i}\right)$. Then

$$
\mathbb{E}\left[\operatorname{frag}\left(R_{n}\right)\right] \geqslant \mathbb{E}\left[\sum_{i=1}^{m} v\left(H_{i}\right) \kappa\left(R_{n}, H_{i}\right)\right]-n_{0} \mathbb{P}\left[\operatorname{big}\left(R_{n}\right) \leqslant n_{0}\right]
$$

Now $(\mathcal{A}, \tau)$ is smooth by Theorem 2.4 (a). Thus as $n \rightarrow \infty$

$$
\mathbb{E}\left[\sum_{i=1}^{m} v\left(H_{i}\right) \kappa\left(R_{n}, H_{i}\right)\right] \rightarrow \alpha
$$

by Lemma 6.2 , and by Lemma 3.1

$$
\mathbb{P}\left[\operatorname{big}\left(R_{n}\right) \leqslant n_{0}\right] \leqslant \mathbb{P}\left[\kappa\left(R_{n}\right) \geqslant n / n_{0}\right] \leqslant \mathbb{P}\left[\operatorname{Po}\left(\frac{\nu}{\lambda}\right) \geqslant n / n_{0}-1\right]=o(1) .
$$

Hence $\mathbb{E}\left[\operatorname{frag}\left(R_{n}\right)\right] \geqslant \alpha-o(1) \geqslant c+1$ for $n$ sufficiently large, contradicting our choice of c. 
Proof of Theorem 2.2 By Lemma 3.4, whp $\operatorname{Frag}\left(R_{n}\right) \in \mathcal{F}_{\mathcal{A}}$. Hence by Lemma 7.2, $(\mathcal{A}, \tau)$ and $(\mathcal{C}, \tau)$ are smooth (this also follows from Theorem $2.4(\mathrm{a}))$ and $F_{n} \rightarrow_{T V} R$ as required. Finally note that $\mathbb{E}[v(F)]$ is $\rho D^{\prime}(\rho, \tau)$, which is finite by the last lemma.

Proof of Corollary 2.3 The only thing that does not follow directly from the fact that $F_{n} \rightarrow_{T V} F$ is the convergence of the moments in part (b) (which yields the results on moments in part (c)). But $0 \leqslant \kappa\left(F_{n}, \mathcal{D}\right) \leqslant \kappa\left(R_{n}\right)-1 \leqslant \operatorname{Po}\left(\frac{\nu}{\lambda}\right)$ in distribution by Lemma 3.1, and so convergence for the $j$ th moment follows from convergence in total variation.

\section{Proof of appearances results}

Theorem 2.5 and Proposition 2.6 may be proved along the lines of the corresponding proofs in [47], but for completeness we give proofs here.

Proof of Theorem 2.5 Let $\alpha=\lambda^{e(H)+1} /\left(2 e^{2} \gamma^{h}(h+2) h\right.$ !). We shall prove that there exists $n_{0}$ such that

$$
\operatorname{Pr}\left[f_{H}\left(R_{n}\right) \leqslant \alpha n\right]<e^{-\alpha n} \quad \text { for all } n \geqslant n_{0} .
$$

We often write $x$ instead of $\lfloor x\rfloor$ or $\lceil x\rceil$ to avoid cluttering up formulae: this should cause the reader no problems. Since $\alpha>0,2^{\alpha} \geqslant(1+\epsilon)^{4}$ for some $0<\epsilon<\frac{1}{2}$. Note that

$$
(1-\epsilon)(1+\epsilon)^{2}>1 \text {. }
$$

Let $g(n)$ denote $\tau\left(\mathcal{A}_{n}\right)$. Since $(\mathcal{A}, \tau)$ has growth constant $\gamma$, there is a positive integer $n_{0}$ such that for each $n \geqslant n_{0}$ we have

$$
(1-\epsilon)^{n} \cdot n ! \gamma^{n} \leqslant g(n) \leqslant(1+\epsilon)^{n} \cdot n ! \gamma^{n} .
$$

Let $\mathcal{B}$ denote the class of graphs in $\mathcal{A}$ such that $f_{H}(G) \leqslant \alpha v(G)$. Assume that equation (34) does not hold for some $n \geqslant n_{0}$; that is, assume that $\tau\left(\mathcal{B}_{n}\right) \geqslant e^{-\alpha n} g(n)$. Let $\delta=\alpha h$. We shall show that

$$
g((1+\delta) n)>(1+\epsilon)^{(1+\delta) n} \cdot[(1+\delta) n] ! \cdot \gamma^{(1+\delta) n},
$$

which will contradict (36) and complete the proof of the theorem.

In order to establish this inequality, we construct graphs $G^{\prime}$ in $\mathcal{A}$ on vertex set $\{1, \ldots,(1+\delta) n\}$ as follows. First we choose a subset of $\delta n$ special vertices $\left(\left(\begin{array}{c}(1+\delta) n \\ \delta n\end{array}\right)\right.$ choices) and a graph $G \in \mathcal{B}$ on the remaining $n$ vertices. By assumption

$$
\tau\left(\mathcal{B}_{n}\right) \geqslant e^{-\alpha n} \cdot g(n) \geqslant e^{-\alpha n}(1-\epsilon)^{n} \gamma^{n} n !
$$

Next we consider the $\delta n$ special vertices. We partition them into $\alpha n$ (unordered) blocks of size $h$. On each block $B$ we put a copy of $H$ such that the increasing bijection from $\{1, \ldots, h\}$ to $B$ is an isomorphism between $H$ and this copy. Call the lowest numbered 
vertex in $B$ the root $r_{B}$ of the block. For each block $B$ we choose a non-special vertex $v_{B}$ and add the edge $r_{B} v_{B}$ between the root and this vertex: observe that $H$ appears at $B$ in $G^{\prime}$. This completes the construction of $G^{\prime}$ : note that $G \in \mathcal{A}$ since $H$ is freely attachable to $\mathcal{A}$. For each choice of special vertices, the weight of constructions is

$$
\begin{aligned}
& \tau\left(\mathcal{B}_{n}\right) \cdot\left(\begin{array}{c}
\delta n \\
h \cdots h
\end{array}\right) \cdot \frac{1}{(\alpha n) !} \cdot n^{\alpha n}\left(\lambda \lambda^{e(H)}\right)^{\alpha n} \\
= & \tau\left(\mathcal{B}_{n}\right) \cdot \frac{(\delta n) ! n^{\alpha n}}{(h !)^{\alpha n}(\alpha n) !}\left(\lambda^{e(H)+1}\right)^{\alpha n} \\
\geqslant & \tau\left(\mathcal{B}_{n}\right) \cdot(\delta n) !\left(\frac{\lambda^{e(H)+1}}{h ! \alpha}\right)^{\alpha n}
\end{aligned}
$$

since $(\alpha n) ! \leqslant \alpha^{\alpha n} n^{\alpha n}$.

How often is the same graph $G^{\prime}$ constructed? Call an oriented edge $e=u v$ good in $G^{\prime}$ if it is a cut-edge in $G^{\prime}$, the component $\tilde{G}$ of $G^{\prime}-e$ containing $u$ has $h$ nodes, $u$ is the least of these nodes, and the increasing map from $\{1, \ldots, h\}$ to $V(\tilde{G})$ is an isomorphism between $H$ and $\tilde{G}$. Observe that each added oriented edge $r_{B} v_{B}$ is good. Indeed, there is exactly one good oriented edge for each appearance of $H$ in $G$. We shall see that $G^{\prime}$ contains at most $(h+2) \alpha n$ good oriented edges. It will then follow that the number of times that $G^{\prime}$ can be constructed is at most $\left(\begin{array}{c}(h+2) \alpha n \\ \alpha n\end{array}\right) \leqslant((h+2) e)^{\alpha n}$.

We may bound the number of good edges in $G^{\prime}$ as follows. (a) There are exactly $\alpha n$ added oriented edges $r_{B} v_{B}$. (b) There are at most $\alpha n$ good oriented edges $e=u v$ in $E(G)$ (that is, such that the unoriented edge is in $G$ ): for in this case the entire component of $G^{\prime}-e$ containing $u$ must be contained in $G$ (if it contained any other vertex it would have more than $h$ vertices), and so the number of them is at most $f_{H}(G)$. (c) There are at most han 'extra' good oriented edges. To see this, consider a block $B$, and let $\tilde{H}$ denote the connected graph formed from the induced subgraph $G^{\prime}[B]$ (which is isomorphic to $H$ ) together with the vertex $v_{B}$ and the edge $r_{B} v_{B}$. Each 'extra' good oriented edge must be a cut edge in such a graph $\tilde{H}$ oriented away from $v_{B}$, and in each graph $\tilde{H}$ there are at most $h$ cut-edges.

We may put the above results together to obtain

$$
\begin{aligned}
g((1+\delta) n) & \geqslant\left(\begin{array}{c}
(1+\delta) n \\
\delta n
\end{array}\right) \cdot e^{-\alpha n}(1-\epsilon)^{n} \gamma^{n} n ! \cdot(\delta n) !\left(\frac{\lambda^{e(H)+1}}{h ! \alpha}\right)^{\alpha n}((h+2) e)^{-\alpha n} \\
& =((1+\delta) n) ! \cdot \gamma^{(1+\delta) n} \cdot(1-\epsilon)^{n} \cdot 2^{\alpha n} \\
& \geqslant g((1+\delta) n)(1+\epsilon)^{-(1+\delta) n} \cdot(1-\epsilon)^{n} \cdot(1+\epsilon)^{4 n} \\
& \geqslant g((1+\delta) n)\left((1-\epsilon)(1+\epsilon)^{2}\right)^{n}>g((1+\delta) n),
\end{aligned}
$$

which is the desired contradiction.

Proof of Proposition 2.6 Denote $v(H)$ by $h$ and $\gamma^{-1}$ by $\rho$. Observe first that

$$
\mathbb{E}\left[X_{n}(H)\right]=\left(\begin{array}{l}
n \\
h
\end{array}\right)(n-h) \lambda \lambda^{e(H)} \frac{\tau\left(\mathcal{A}_{n-h}\right)}{\tau\left(\mathcal{A}_{n}\right)} \sim \lambda n \frac{\rho^{h} \lambda^{e(H)}}{h !} .
$$


Now consider $\mathbb{E}\left[\left(X_{n}(H)\right)_{2}\right]$. For each graph $G$ on $\{1, \ldots, n\}$ let $Y_{1}(G, H)$ be the number of ordered pairs of appearances in $G$ of $H$ with disjoint vertex sets and such that the roots are not adjacent; and let $Y_{2}(G, H)$ be the number of ordered pairs of appearances in $G$ of $H$ such that either the vertex sets meet or the roots are adjacent. Thus $\left(X_{n}(H)\right)_{2}=$ $Y_{1}\left(R_{n}, H\right)+Y_{2}\left(R_{n}, H\right)$. Now

$$
\mathbb{E}\left[Y_{1}\left(R_{n}, H\right)\right]=\frac{(n)_{2 h}}{(h !)^{2}}(n-2 h)^{2} \lambda^{2} \lambda^{2 e(H)} \frac{\tau\left(\mathcal{A}_{n-2 h}\right)}{\tau\left(\mathcal{A}_{n}\right)} \sim\left(\lambda n \frac{\rho^{h} \lambda^{e(H)}}{h !}\right)^{2} .
$$

But a graph $G$ of order at most $2 h$ either consists of two appearances of $H$ with adjacent roots (and then $G$ has exactly two appearances of $H$ ), or the number of appearances of $H$ is at most the number of bridges in $G$. Thus $Y_{2}(G, H)$ is at most $2 h$ times the number of components of $G$ of order at most $2 h$, which is at most $2 h \kappa(G)$; and so

$$
\mathbb{E}\left[Y_{2}\left(R_{n}, H\right)\right] \leqslant 2 h \mathbb{E}\left[\kappa\left(R_{n}\right)\right] \leqslant 2 h\left(1+\frac{\nu}{\lambda}\right)
$$

since $\mathbb{E}\left[\kappa\left(R_{n}\right)\right] \leqslant 1+\frac{\nu}{\lambda}$ by Lemma 3.1. Hence

$$
\mathbb{E}\left[\left(X_{n}(H)\right)_{2}\right]=\mathbb{E}\left[Y_{1}\left(R_{n}, H\right)\right]+O(1) \sim\left(\lambda n \frac{\rho^{h} \lambda^{e(H)}}{h !}\right)^{2} .
$$

Thus the variance of $X_{n}(H)$ is $o\left(\mathbb{E}\left[\left(X_{n}(H)\right)^{2}\right]\right)$, and the result follows by Chebyshev's inequality.

Finally consider the remark concerning disjoint pendant appearances following Proposition 2.6. By the above proof, it suffices to note that in any graph $G$ the number of pendant appearances of $H$ that share a vertex or edge with some other pendant appearance is at most $Y_{2}(G, H)$, and so $\mathbb{E}\left[\tilde{X}_{n}(H)\right] \leqslant \mathbb{E}\left[Y_{2}\left(R_{n}, H\right)\right]=O(1)$.

\section{Concluding remarks}

Sometimes it may be helpful to generalise our probability model one step further. Bridges play a major role in this work. Recall that a bridge in a graph $G$ is an edge $e$ such that $G-e$ has one more component than $G$. For a graph $G$ we let $e_{0}(G)$ be the number of bridges in $G$ (the 0 is since a bridge is in 0 cycles) and let $e_{1}(G)=e(G)-e_{0}(G)$. In the definition of $\tau(G)$ let us replace $\lambda^{e(G)}$ by $\lambda_{0}^{e_{0}(G)} \lambda_{1}^{e_{1}(G)}$, where $\lambda_{0}$ and $\lambda_{1}$ are the edge-parameters.

Thus the distribution of the random graph $R_{n} \in_{\tau} \mathcal{A}$ is as follows. Let $\lambda_{0}>0$, $\lambda_{1}>0$ and $\nu>0$, let $\lambda=\left(\lambda_{0}, \lambda_{1}\right)$, and let $\tau=(\lambda, \nu)$. For each graph $G$ we let $\lambda^{e(G)}$ denote $\lambda_{0}^{e_{0}(G)} \lambda_{1}^{e_{1}(G)}$, and let $\tau(G)=\lambda^{e(G)} \nu^{\kappa(G)}$. Now we proceed as before, and let $\mathbb{P}\left(R_{n}=G\right) \propto \tau(G)$ for each $G \in \mathcal{A}_{n}$. The most natural and interesting case is when $\lambda_{0}=\lambda_{1}$ but we learn more about the role of bridges by allowing the edge-parameters to differ. 
The results and proofs above change in a predictable way. We simply replace $\lambda$ by $\lambda_{0}$, except when $\lambda$ appears as $\lambda^{e(G)}$, which we now interpret as $\lambda_{0}^{e_{0}(G)} \lambda_{1}^{e_{1}(G)}$. This holds even for Proposition 2.6, where $\lambda \cdot \lambda^{e(H)}$ becomes $\lambda_{0} \cdot \lambda_{0}^{e_{0}(H)} \lambda_{1}^{e_{1}(H)}$.

There are two places where the change is most apparent. Theorem 2.4 is the upside, where the role of bridges is brought out: each $\lambda$ is replaced by $\lambda_{0}$, and in particular everything depends on how $\gamma$ compares to $\lambda_{0} e$. Lemma 2.7 is the downside: we noted that the previous proofs that the classes in parts (b) and (c) had growth constants in the uniform case extended easily to yield growth constants in the weighted case, but that holds only when $\lambda_{0}=\lambda_{1}$. Can we drop this extra condition?

In the addable minor-closed case we could easily introduce more edge-weights, though it is not clear how much more we would learn. For example, given a graph $G$ and an edge $e=u v$ in $G$, we could let $f(e)$ be the maximum number of edge-disjoint paths between $u$ and $v$ in $G-e$; let $e_{k}(G)$ be the number of edges $e$ in $G$ with $f(e)=k$; and let $\lambda^{e(G)}$ mean $\prod_{k \geqslant 0} \lambda_{k}^{e_{k}(G)}$, where each parameter $\lambda_{k}>0$. Then the results above for an addable minor-closed class still hold, with the same proofs - and perhaps we do learn something? Indeed, we could go as far as the very general model in [50], as long as we ensure that $\log \tau(G)=O(v(G))$.

Acknowledgement I would like to thank Kerstin Weller and the referee for helpful comments.

\section{References}

[1] J.P. Bell and S.N. Burris. Asymptotics for logical limit laws; when the growth of the components is in an RT class. Trans. Amer. Math. Soc 355 (2003) $3777-3794$.

[2] E.A. Bender, E.R. Canfield and L.B. Richmond. Coefficients of functional compositions often grow smoothly. Electron. J. Combin. 15 (2008) \#R21.

[3] E. Bender and Z. Gao. Asymptotic enumeration of labelled graphs with a given genus. Electron. J. Combin. 18 (2011) \#P13.

[4] E. Bender, Z. Gao and N. Wormald. The number of labeled 2-connected planar graphs. Electron. J. Combin. 9 (2002) \#R43.

[5] J. van den Berg and J.E. Steif. Percolation and the hard-core lattice gas model. Stoch. Proc. Appl. 49 (1994) 179 - 197.

[6] O. Bernardi, M. Noy and D. Welsh. Growth constants of minor-closed classes of graphs. J. Combin. Theory B 100 (2010) 468 - 484.

[7] N. Bernasconi, K. Panagiotou and A. Steger. On properties of random dissections and triangulations. in Proc SODA 2008, 132 - 141.

[8] N. Bernasconi, K. Panagiotou and A. Steger. On the degree sequence of random outerplanar and series-parallel graphs. LNCS 5171 (2008) 303 - 316.

[9] N. Bernasconi, K. Panagiotou and A. Steger. The degree sequence of random graphs from subcritical classes. Combin. Prob. Comput. 18 (2009) 647 - 681. 
[10] M. Bodirsky, E. Fusy, M. Kang and S. Vigerske. Boltzmann samplers, Pólya theory, and cycle pointing. SIAM J. Comput. 40 (2011) $721-769$.

[11] M. Bodirsky, O. Giménez, M. Kang and M. Noy. On the number of series-parallel and outerplanar graphs. Proceedings of European Conference on Combinatorics, Graph Theory, and Applications (EuroComb 2005), Discrete Math. Theor. Comput. Sci. Proc. Vol. AE (2005) $383-388$.

[12] M. Bodirsky, O. Giménez, M. Kang and M. Noy. Enumeration and limit laws for series-parallel graphs. European Journal of Combinatorics 28 (2007) 2091 - 2105.

[13] M. Bodirsky, M.L. Löffler, M. Kang and C. McDiarmid. Random cubic planar graphs. Random Structures Algorithms 30 (2007) 78 - 94.

[14] B. Bollobás. Random Graphs. Second Edition, Cambridge University Press, 2001.

[15] S.N. Burris. Number theoretic density and logical limit laws. Mathematical Surveys and Monographs 86, AMS, 2001.

[16] G. Chapuy, E. Fusy, O. Giménez and M. Noy. On the diameter of random planar graphs. Proceedings of the 21st International Meeting on Probabilistic, Combinatorial, and Asymptotic Methods in the Analysis of Algorithms (AofA'10), DMTCS Proceedings, vol.AM (2010) 65-78.

[17] G. Chapuy, E. Fusy, O. Giménez, B. Mohar and M. Noy. Asymptotic enumeration and limit laws for graphs of fixed genus. J. Combinatorial Theory Ser A 118 (2011) $748-777$.

[18] G. Chapuy, E. Fusy, M. Kang and B. Shoilekova. A complete grammar for decomposing a family of graphs into 3-connected components. Electronic J. Combinatorics 15 (2008) \#R148.

[19] R. Diestel. Graph Theory. Fourth Edition, Springer-Verlag, Heidelberg, 2010.

[20] C. Dowden. The evolution of uniform random planar graph. Electronic J. Combinatorics 17 (2010) 1 - 20.

[21] C. Dowden. Random planar graphs with bounds on the maximum and minimum degrees. Graphs and Combinatorics 27 (2011) 87 - 107.

[22] M. Drmota, E. Fusy, M. Kang, V. Kraus and J. Rué. Asymptotic study of subcritical graph classes. SIAM J. Discrete Mathematics 25 (2011), 1615-1651.

[23] M. Drmota, O. Giménez and M. Noy. Vertices of given degree in series-parallel graphs. Random Struct. Algorithms 36 (2010) 273-314.

[24] M. Drmota, O. Giménez and M. Noy. Degree distribution in random planar graphs. J. Comb. Theory, Ser. A 118 (2011) 2102-2130.

[25] M. Drmota, O. Giménez and M. Noy. The Maximum Degree of Series-Parallel Graphs. Combinatorics, Probability \&5 Computing 20 (2011) 529-570.

[26] M. Drmota, O. Giménez, M. Noy, K. Panagiotou and A. Steger. The maximum degree of random planar graphs. Proceedings of the 23rd Annual ACM-SIAM Symposium on Discrete Algorithms (SODA '12), 2012, 281-287. 
[27] Z. Dvorák and S. Norine. Small graph classes and bounded expansion. J. Combinatorial Theory B 100 (2010) 171 - 175.

[28] P. Flajolet and R. Sedgewick. Analytic Combinatorics. CUP, 2009.

[29] N. Fountoulakis and K. Panagiotou. 3-connected cores in random planar graphs. Combinatorics, Probability \& Computing 20 (2011) 381 - 412.

[30] E. Fusy. Uniform random sampling of planar graphs in linear time. Random Structures and Algorithms 35 (2009) $464-522$.

[31] S. Gerke, O. Giménez, M. Noy and A. Weißl. The number of graphs not containing $K_{3,3}$ as a minor. Electron. J. Combin. 15 (2008) R114.

[32] S. Gerke and C. McDiarmid. On the number of edges in random planar graphs. Combinatorics, Probability and Computing 13 (2004) 165 - 183.

[33] S. Gerke, C. McDiarmid, A. Steger and A. Weissl. Random planar graphs with n nodes and a fixed number of edges. Proceedings of the ACM-SIAM Symposium on Discrete Algorithms (SODA) 2005, 999 - 1007.

[34] S. Gerke, C. McDiarmid, A. Steger and A. Weissl. Random planar graphs with given average degree. in Combinatorics, Complexity and Chance, a tribute to Dominic Welsh (G. Grimmett and C. McDiarmid eds) Oxford University Press, 2007, 83 102.

[35] O. Giménez and M. Noy. Asymptotic enumeration and limit laws of planar graphs. J. Amer. Math. Soc. 22 (2009) 309-329.

[36] O. Giménez and M. Noy. Counting planar graphs and related families of graphs. in Surveys in Combinatorics 2009, 169 - 329, Cambridge University Press, Cambridge, 2009.

[37] O. Giménez, M. Noy and J. Rué. Graph classes with given 3-connected components: asymptotic counting and critical phenomena. Electronic Notes in Discrete Mathematics 29 (2007) 521 - 529.

[38] G. Grimmett. The Random-Cluster Model. Springer, 2006.

[39] S. Janson, T. Łuczak and A. Ruciński. Random Graphs. Wiley Interscience, 2000.

[40] M. Kang and M. Loebl. The enumeration of planar graphs via Wick's theorem. Advances in Mathematics 221 (2009) 1703 - 1724.

[41] M. Kang and T. Łuczak. Two critical periods in the evolution of random planar graphs. Trans. Amer. Math. Soc 364 (2012), 4239-4265.

[42] M. Kang and C. McDiarmid. Random unlabelled graphs containing few disjoint cycles. Random Structures and Algorithms 38 (2011) $174-204$.

[43] V. Kurauskas and C. McDiarmid. Random graphs with few disjoint cycles. Combinatorics, Probability and Computing 20 (2011) 763 - 775.

[44] V. Kurauskas and C. McDiarmid. Random graphs containing few disjoint excluded minors. Random Structures and Algorithms, published online 27 July 2012. 
[45] J.H. van Lint and R.M. Wilson. A Course in Combinatorics. 2nd ed. Cambridge University Press, Cambridge, 2001.

[46] W. Mader. Homomorphiesätze für Graphen. Math. Ann. (1968) $178154-168$.

[47] C. McDiarmid. Random graphs on surfaces. J. Combinatorial Theory B 98 (2008) $778-797$.

[48] C. McDiarmid. Random graphs from a minor-closed class. Combinatorics, Probability and Computing 18 (2009) 583 - 599.

[49] C. McDiarmid. On graphs with few disjoint $t$-star minors. European J. Comb. 32 (2011) $1394-1406$.

[50] C. McDiarmid. Connectivity for random graphs from a weighted bridge-addable class, 2012, http://arxiv.org/abs/1203.3398.

[51] C. McDiarmid and B. Reed. On the maximum degree of a random planar graph. Combinatorics, Probability and Computing 17 (2008) 591- 601.

[52] C. McDiarmid, A. Steger and D. Welsh. Random planar graphs. J. Combinatorial Theory B 93 (2005) 187 - 206.

[53] C. McDiarmid, A. Steger and D. Welsh. Random graphs from planar and other addable classes. Topics in Discrete Mathematics (M. Klazar, J. Kratochvil, M. Loebl, J. Matousek, R. Thomas, P. Valtr eds), Algorithms and Combinatorics 26, Springer, 2006, $231-246$.

[54] J.W. Moon. Counting labelled trees. Canadian Mathematical Monographs 1, 1970.

[55] S. Norine, P. Seymour, R. Thomas and P. Wollan. Proper minor-closed families are small. J. Combin. Theory B 96 (2006) $754-757$.

[56] K. Panagiotou and A. Steger. Maximal biconnected subgraphs of random planar graphs. ACM Transactions on Algorithms 6 (2010) art. no. 31.

[57] K. Panagiotou and A. Steger. On the degree sequence of random planar graphs. Proceedings of the 22nd Annual ACM-SIAM Symposium on Discrete Algorithms (SODA '11), 2011, 1198-1210.

[58] N. Robertson and P.D. Seymour. Graph minors I - XX. J. Combin. Theory B (1983 $-2004)$.

[59] R.P. Stanley. Enumerative Combinatorics, Vol 2. Cambridge University Press, 1999. 